\title{
Equivariant Ricci flow with surgery and applications to finite group actions on geometric 3-manifolds
}

\author{
JONATHAN DINKELBACH \\ BERNHARD LEEB
}

\begin{abstract}
We apply an equivariant version of Perelman's Ricci flow with surgery to study smooth actions by finite groups on closed 3-manifolds. Our main result is that such actions on elliptic and hyperbolic 3-manifolds are conjugate to isometric actions. Combining our results with results by Meeks and Scott [17], it follows that such actions on geometric 3-manifolds (in the sense of Thurston) are always geometric, ie there exist invariant locally homogeneous Riemannian metrics. This answers a question posed by Thurston [32].
\end{abstract}

57M60, 57M50; 53C21, 53C44

\section{Introduction}

The main results of this paper concern smooth group actions on geometric 3-manifolds:

Theorem E (Actions on elliptic manifolds are standard.) Any smooth action by a finite group on an elliptic 3-manifold is smoothly conjugate to an isometric action.

Theorem H (Actions on closed hyperbolic manifolds are standard.) Any smooth action by a finite group on a closed hyperbolic 3-manifold is smoothly conjugate to an isometric action.

We also show (Theorem 5.6) that smooth actions by finite groups on closed $\left(S^{2} \times \mathbb{R}\right)$ manifolds are geometric, ie there exist invariant Riemannian metrics locally isometric to $S^{2} \times \mathbb{R}$. See Meeks and Yau [18] for earlier results concerning this case.

Corresponding results for the other five 3-dimensional Thurston geometries had been obtained by Meeks and Scott [17]. Combining our results with theirs it follows that smooth actions by finite groups on closed geometric 3-manifolds are always geometric, ie there exist invariant locally homogeneous Riemannian metrics. This answers a question posed by Thurston [32]. 
Theorem $\mathrm{H}$ extends to actions on compact 3-manifolds with boundary whose interior admits a complete hyperbolic structure of finite volume (Theorem 5.5).

Theorems $\mathrm{E}$ and $\mathrm{H}$ have been previously known in most cases. For free actions, they are due to Perelman in his fundamental work on the Ricci flow in dimension three $[25 ; 27 ; 26]$. For orientation preserving nonfree actions, they have been proven by Boileau, Leeb and Porti [2] along the lines suggested by Thurston [31] and based on Thurston's Hyperbolization Theorem for Haken manifolds, using techniques from 3-dimensional topology, the deformation theory of geometric structures and the theory of metric spaces with curvature bounded below. They have also been known in several cases for orientation nonpreserving nonfree actions.

In this paper we give a unified approach by applying the Ricci flow techniques to the case of nonfree actions. This also provides an alternative proof in the orientation preserving nonfree case. Our argument is based on several deep recent results concerning the Ricci flow with cutoff on closed 3-manifolds, namely its long time existence for arbitrary initial metrics (see Perelman [27], Kleiner and Lott [15], Morgan and Tian [21] and Bamler [1]), its extinction in finite time on nonaspherical prime 3-manifolds (see Perelman [26], Colding and Minicozzi [5; 6] and Morgan and Tian [21]) and, for Theorem H, the analysis of its asymptotic long time behavior (see Perelman [27] and Kleiner and Lott [15]).

Given a smooth action $\rho: G \curvearrowright M$ by a finite group on a closed 3-manifold, and given any $\rho$-invariant initial Riemannian metric $g_{0}$ on $M$, there is no problem in running an equivariant Ricci flow with cutoff, because the symmetries are preserved between surgery times and can be preserved while performing the surgeries. During the flow, the underlying manifold and the action may change. In order to understand the initial action $\rho$, one needs to compare the actions before and after a surgery, and to verify that, short before the extinction of connected components, the actions on them are standard. The main issue to be addressed here is that the caps occurring in highly curved regions close to the singularities of the Ricci flow may have nontrivial stabilizers whose actions one has to control. Since on an elliptic 3-manifold the Ricci flow goes extinct in finite time for any initial metric, one can derive Theorem E. If $M$ is hyperbolic, its hyperbolic metric is unique up to diffeomorphisms by Mostow rigidity. It turns out that for large times the time slice is (again) diffeomorphic to $M$ and the Riemannian metric produced by the Ricci flow converges smoothly to the hyperbolic metric up to scaling and diffeomorphisms. This leads to Theorem H. Our methods apply also to actions on the nonirreducible prime 3 -manifold $S^{2} \times S^{1}$ and its quotient manifolds because the Ricci flow goes extinct in this case, too. 
Acknowledgements We are grateful to Richard Bamler for many useful comments on an earlier version of this article. We thank the Deutsche Forschungsgemeinschaft (DFG-project LE 1312/1) and the Hausdorff Research Institute for Mathematics in Bonn for financial support.

\section{Topological preliminaries}

\subsection{Standard actions on geometric 3-manifolds}

Throughout this paper, we consider smooth actions by finite groups on 3-manifolds. As eg in Meeks and Scott [17], we call an action $\rho: G \curvearrowright M^{3}$ on a closed connected 3 -manifold standard if it preserves a geometric structure in the sense of Thurston [29; 33 ], ie if there exists an invariant locally homogeneous Riemannian metric. Note that this requires the manifold itself to be geometric and the type of geometric structure is uniquely determined.

In the case of the elliptic and hyperbolic geometries the geometric structure on $M$ is unique up to diffeomorphism. (In the elliptic case, this follows from the isometry classification of elliptic manifolds (see eg Thurston [33, Theorem 4.4.14]) and the topological classification of lens spaces by Brody [3] (see also Hatcher [13, Theorem 2.5]). In the hyperbolic case, this is a consequence of Mostow rigidity [22].) This means that an action on an elliptic or on a closed hyperbolic 3-manifold is standard if and only if it is smoothly conjugate to an isometric action.

Sometimes, we will need to consider actions on a few simple noncompact manifolds or compact manifolds with nonempty boundary: We call an action on the (open or closed) unit ball standard, if it is smoothly conjugate to an orthogonal action. We call an action on the round cylinder $S^{2} \times \mathbb{R}$, or on one of the orientable $\mathbb{Z}_{2}$-quotients $S^{2} \times \mathbb{Z}_{2} \mathbb{R} \cong \mathbb{R} P^{3}-\bar{B}^{3}$ and $S^{2} \times \mathbb{Z}_{2}[-1,1] \cong \mathbb{R} P^{3}-B^{3}$ standard, if it is smoothly conjugate to an isometric action (say, with respect to the $\left(S^{2} \times \mathbb{R}\right)$-structure).

If $M$ is disconnected, we call an action $G \curvearrowright M$ standard if for each connected component $M_{0}$ of $M$ the restricted action $\operatorname{Stab}_{G}\left(M_{0}\right) \curvearrowright M_{0}$ is standard.

\subsection{Equivariant diffeomorphisms of the 2-sphere are standard}

We need the following equivariant version of a result of Munkres [23] regarding isotopy classes of diffeomorphisms of the 2 -sphere.

Proposition 2.1 Let $\rho: H \curvearrowright S^{2}$ be an orthogonal action of a finite group on the 2-dimensional unit sphere. Then every $\rho$-equivariant diffeomorphism $S^{2} \rightarrow S^{2}$ is $\rho$-equivariantly isotopic to an isometric one. 
Equivalently, in terms of quotient orbifolds:

Proposition 2.2 Any diffeomorphism $\phi: \mathcal{O}_{1} \rightarrow \mathcal{O}_{2}$ of closed spherical 2-orbifolds is isotopic to an isometry.

We recall that a diffeomorphism of orbifolds is a homeomorphism which locally lifts to an equivariant diffeomorphism of orbifold charts. In particular, it maps the singular locus to the singular locus and preserves the types of singular points.

Proof For $\mathcal{O}_{1} \cong \mathcal{O}_{2} \cong S^{2}$ the result is proven by Munkres [23] (see also Thurston [33, Theorem 3.10.11]) and for $\mathbb{R} P^{2}$ in Epstein [8, Theorem 5.5]. We therefore assume that the orbifolds have singularities and extend the result to this case using standard arguments from 2-dimensional topology.

Let us first recall the list of nonsmooth closed spherical 2-orbifolds. By a cone point, we mean an isolated singular point.

- The 2-sphere with two or three cone points, and the projective plane with one cone point.

- The closed 2-disk with reflector boundary, at most three corner reflector points and at most one cone point in the interior. (The cone point can only occur in the case of at most one corner reflector and must occur if there is precisely one corner reflector.)

From the classification we observe that a closed spherical 2-orbifold is determined up to isometry by its underlying surface and the types of the singular points. Hence $\mathcal{O}_{1}$ and $\mathcal{O}_{2}$ must be isometric. After suitably identifying them, we may regard $\phi$ as a self-diffeomorphism of a closed spherical 2 -orbifold $\mathcal{O}$ which fixes every cone point and corner reflector. We can arrange moreover that $\phi$ preserves orientation if $\mathcal{O}$ is orientable, and locally preserves orientation near the cone point if $\mathcal{O}$ is a projective plane with one cone point. It is then a consequence that, when the singular locus is one-dimensional, ie when $\mathcal{O}$ has reflector boundary, $\phi$ preserves every singular edge (and circle) and acts on it as an orientation preserving diffeomorphism.

Lemma 2.3 Let $D=D(1)$ be the open unit disk and let $D^{\prime}=D\left(r^{\prime}\right), 0<r^{\prime} \leq 1$, be a round subdisk centered at the origin. Suppose that $\rho: H \curvearrowright D$ is an orthogonal action of a finite group, and that $\phi: D^{\prime} \rightarrow D$ is an orientation preserving $\rho$-equivariant smooth embedding fixing 0 . Then $\phi$ is isotopic, via a compactly supported $\rho$-equivariant isotopy, to a $\rho$-equivariant smooth embedding $D^{\prime} \rightarrow D$ which equals $\pm \operatorname{id}_{D^{\prime}}$ near 0 and, if $\rho$ preserves orientation, $+\mathrm{id}_{D^{\prime}}$. 
Proof (Compare eg [33, end of proof of Lemma 3.10.12]). We may first isotope $\phi$ to make it linear near 0 by interpolating with its differential $d \phi_{0}$ at 0 . Indeed, using a rotationally symmetric smooth test function $\theta$ on $D^{\prime}$, we put

$$
\phi_{t}:=\phi+t \theta_{\lambda}\left(d \phi_{0}-\phi\right) \quad 0 \leq t \leq 1
$$

where $\theta_{\lambda}(x):=\theta(x / \lambda)$. Then $\left\|d \phi_{t}-d \phi\right\| \leq C \lambda t$, and for sufficiently small $\lambda>0$ we obtain an isotopy.

Assume now that $\phi$ agrees near 0 with an orientation preserving $\rho$-equivariant linear map $A$. If $\rho(H)$ contains reflections, then $A$ preserves a line and, if $\rho(H)$ also contains a rotation of order $\geq 3$, is a dilation. One can equivariantly isotope $\phi$ to make it equal to \pm id near 0 . If $\rho(H)$ preserves orientation, then $A$ is a homothety if $|\rho(H)| \geq 3$, and an arbitrary orientation preserving linear automorphism otherwise. In both cases, one can equivariantly isotope $\phi$ to make it equal to id near 0 .

Due to Lemma 2.3, we may assume after applying a suitable isotopy, that $\phi$ equals the identity in a neighborhood of every cone point and every corner reflector. By sliding along the singular edges, we may isotope $\phi$ further so that it fixes the singular locus pointwise. Since $\phi$ preserves orientation, if $\mathcal{O}$ has boundary reflectors, an argument as in the proof of Lemma 2.3 (but simpler) allows to isotope $\phi$ so that it fixes a neighborhood of the singular locus pointwise.

Lemma 2.4 (i) Suppose that $\mathcal{O}$ is a 2 -sphere with two or three cone points and that $\phi: \mathcal{O} \rightarrow \mathcal{O}$ is an orientation preserving diffeomorphism which fixes every cone point. Then $\phi$ is isotopic to the identity.

(ii) Suppose that $D$ is a closed 2-disk with at most one cone point and that $\phi: D \rightarrow D$ is an orientation preserving diffeomorphism fixing a neighborhood of $\partial D$ pointwise. (It must fix the cone point if there is one.) Then $\phi$ is isotopic to the identity by an isotopy supported on the interior of $D$.

(iii) Suppose that $M$ is a closed Möbius band and that $\phi: M \rightarrow M$ is a diffeomorphism fixing a neighborhood of $\partial M$ pointwise. Then $\phi$ is isotopic to the identity by an isotopy supported on the interior of $M$.

Remark 2.5 In case (i) and in case (ii) when there is a cone point, the isotopy will in general not be supported away from the cone points but must rotate around them (Dehn twists!).

Proof (i) It suffices to consider the case of three cone points. Let us denote them by $p, q$ and $r$. We choose a smooth arc $\gamma$ from $p$ to $q$ avoiding $r$. We may assume 
that the image arc $\phi(\gamma)$ is transversal to $\gamma$. Let $x_{1}, \ldots, x_{n}, n \geq 0$, denote the interior intersection points of $\gamma$ and $\phi(\gamma)$ numbered according to their order along $\phi(\gamma)$. Set $x_{0}=p$ and $x_{n+1}=q$.

Suppose that the number $n$ of transverse intersections cannot be decreased by isotoping $\phi$, and that $n \geq 1$. For $i=0$ and $n$, we consider the subarc $\alpha_{i}$ of $\phi(\gamma)$ from $x_{i}$ to $x_{i+1}$. Let $\beta_{i}$ be the subarc of $\gamma$ with the same endpoints. We denote by $D_{i}$ the disk bounded by the circle $\alpha_{i} \cup \beta_{i}$ whose interior is disjoint from $\gamma$, ie such that $p \notin D_{n}$ and $q \notin D_{0}$. This choice implies that the disks $D_{0}$ and $D_{n}$ have disjoint interiors, because $\partial D_{0} \cap \operatorname{int}\left(D_{n}\right)=\varnothing$ and $\partial D_{n} \cap \operatorname{int}\left(D_{0}\right)=\varnothing$. (Note however, that they may contain other subarcs of $\phi(\gamma)$.) It follows that at least one of the disks $D_{0}$ and $D_{n}$ does not contain the third cone point $r$. We then can push this disk through $\gamma$ by applying a suitable isotopy of $\phi$ and thereby reduce the number of intersection points, a contradiction. (Obviously, we can do the same if there are just two cone points $p$ and $q$.

This shows that we can isotope $\phi$ such that $\phi(\gamma)$ and $\gamma$ have no interior intersection points at all. We may isotope further so that $\phi(\gamma)=\gamma$ and, since $\phi$ preserves orientation, even so that $\phi$ fixes a neighborhood of $\gamma$ pointwise; cf Lemma 2.3. This reduces our assertion to case (ii).

(ii) Suppose first that $D$ has a cone point $p$. We then proceed as in case (i). This time we choose $\gamma$ to connect a point on $\partial D$ to $p$. If $\phi(\gamma)$ intersects $\gamma$ transversally, we can remove all intersection points by applying suitable isotopies supported away from $\partial D$. Since $\phi$ preserves orientation, we can isotope $\phi$ such that it equals the identity in a neighborhood of $\partial D \cup \gamma$. This reduces our assertion to the case of a smooth disk. In this case, the result is proven by Munkres [23, Theorem 1.3] (see also Thurston [33, end of proof of Theorem 3.10.11]).

(iii) This fact is proven by Epstein [8, Theorem 3.4].

The claim of Proposition 2.2 now follows from part (i) of Lemma 2.4 if $\mathcal{O}$ is a sphere with two or three cone points, from part (iii) if $\mathcal{O}$ is a projective plane with one cone point, and from part (ii) if $\mathcal{O}$ has boundary reflectors. Note that we have to use isotopies which rotate around cone points. Isotopies supported away from the singular locus do not suffice.

This concludes the proof of Proposition 2.2.

We will use the following consequences of Proposition 2.1 in Section 5. 
Corollary 2.6 (i) Given two isometric actions $\rho_{1}, \rho_{2}: H \curvearrowright \bar{B}^{3}(1)$ of a finite group $H$ on the closed Euclidean unit ball, any $\left(\rho_{1}, \rho_{2}\right)$-equivariant diffeomorphism $\alpha: \partial B \rightarrow$ $\partial B$ extends to a $\left(\rho_{1}, \rho_{2}\right)$-equivariant diffeomorphism $\hat{\alpha}: \bar{B} \rightarrow \bar{B}$.

(ii) The same assertion with $\bar{B}^{3}(1)$ replaced by the complement $\mathbb{R} P^{3}-B^{3}$ of an open round ball with radius $<\pi / 2$ in projective 3 -space equipped with the standard spherical metric.

Proof In both cases we can choose a collar neighborhood $C$ of the boundary sphere and use Proposition 2.1 to extend $\alpha$ to a $\left(\rho_{1}, \rho_{2}\right)$-equivariant diffeomorphism $C \rightarrow C$, which is isometric on the inner boundary sphere. It is then trivial to further extend $\alpha$ equivariantly and isometrically to the rest of the manifold.

Corollary 2.7 Let $\rho: H \curvearrowright S^{2} \times S^{1}$ be a smooth action of a finite group which preserves the foliation $\mathcal{F}$ by the $2-$ spheres $S^{2} \times\{\mathrm{pt}\}$. Then $\rho$ is standard.

We recall that, as defined in Section 2.1, an action on $S^{2} \times S^{1}$ is standard if and only if there exists an invariant Riemannian metric locally isometric to $S^{2} \times \mathbb{R}$.

Proof Since $\rho$ preserves $\mathcal{F}$, it induces an action $\bar{\rho}: H \curvearrowright S^{1}$. We denote the kernel of $\bar{\rho}$ by $H_{0}$.

There exists a $\bar{\rho}$-invariant metric on $S^{1}$. We choose a finite $\bar{\rho}$-invariant subset $A \subset S^{1}$ as follows. If $\bar{\rho}$ acts by rotations, let $A$ be an orbit. Otherwise, if $\bar{\rho}$ acts as a dihedral group, let $A$ be the set of all fixed points of reflections in $\bar{\rho}(H)$. Let $g_{0}$ be a $\rho-$ invariant spherical metric on the union $\Sigma \subset S^{2} \times S^{1}$ of the $\mathcal{F}$-leaves corresponding to the points in $A$.

There exists a $\rho$-invariant line field $\mathcal{L}$ transversal to the foliation $\mathcal{F}$. Following the integral lines of $\mathcal{L}$ we obtain $H_{0}$-equivariant self-diffeomorphisms of $\Sigma$. Using Proposition 2.1, we can modify $\mathcal{L}$ so that these self-diffeomorphisms become $g_{0}-$ isometric. Then a $\rho$-invariant metric locally isometric to $S^{2}(1) \times \mathbb{R}$ can be chosen so that the $\mathcal{F}$-leaves are totally geodesic unit spheres and $\mathcal{L}$ is the line field orthogonal to $\mathcal{F}$.

Remark 2.8 We will show later (see Theorem 5.6) that the same conclusion holds without assuming that $\mathcal{F}$ is preserved by the action. 


\subsection{Equivariant connected sum (decomposition)}

We fix a finite group $G$ and consider smooth actions $\rho$ : $G \curvearrowright M$ on closed (not necessarily connected) 3-manifolds.

Suppose that we are given a $\rho$-invariant finite family of pairwise disjoint embedded 2-spheres $S_{i}^{2} \subset M$. Cutting $M$ along $\bigcup_{i} S_{i}$ yields a compact manifold $\check{M}$ with boundary. To every sphere $S_{i}$ correspond two boundary spheres $S_{i 1}$ and $S_{i 2}$ of $\breve{M}$. The action $\rho$ induces a smooth action $\check{\rho}$ : $G \curvearrowright \breve{M}$. Let $G_{i}:=\operatorname{Stab}_{G}\left(S_{i 1}\right)=$ $\operatorname{Stab}_{G}\left(S_{i 2}\right)$. For every boundary sphere $S_{i j}$ we choose a copy $\bar{B}_{i j}$ of the closed unit 3-ball and an orthogonal action $\check{\rho}_{i j}: G_{i} \curvearrowright B_{i j}$ such that there exists a $G_{i}$-equivariant diffeomorphism $\phi_{i j}: \partial B_{i j} \stackrel{\cong}{\rightarrow} S_{i j}$. We attach the balls $\bar{B}_{i j}$ to $\breve{M}$ using the $\phi_{i j}$ as gluing maps and obtain a closed manifold $M^{\prime}$. The action $\check{\rho}$ extends to a smooth action $\rho^{\prime}: G \curvearrowright M^{\prime}$, and the smooth conjugacy class of $\rho^{\prime}$ does not depend on the choice of the gluing maps $\phi_{i j}$; compare Corollary 2.6 (i). We call $\rho^{\prime}$ an equivariant connected sum decomposition of $\rho$. (Note that the spheres $S_{i}$ are allowed to be nonseparating.)

This construction is reversed by the equivariant connected sum operation. Suppose that $\mathcal{P}=\left\{P_{i}: i \in I\right\}$ is a finite $G$-invariant family of pairwise disjoint two point subsets $P_{i}=\left\{x_{i}, y_{i}\right\}, x_{i} \neq y_{i}$, of $M$. Then there are induced actions of $G$ on $\mathcal{P}$ and on $\bigcup_{i \in I} P_{i}$. Let $G_{i}:=\operatorname{Stab}_{G}\left(x_{i}\right)=\operatorname{Stab}_{G}\left(y_{i}\right)$. We suppose that for every $i \in I$ the actions $d \rho_{x_{i}}: G_{i} \curvearrowright T_{x_{i}} M$ and $d \rho_{y_{i}}: G_{i} \curvearrowright T_{y_{i}} M$ are equivalent via a linear isomorphism $\alpha_{x_{i}}: T_{x_{i}} M \rightarrow T_{y_{i}} M$, respectively, $\alpha_{y_{i}}=\alpha_{x_{i}}^{-1}: T_{y_{i}} M \rightarrow T_{x_{i}} M$. More than that, we require that the family of the $\alpha$ 's is $G$-equivariant, ie if $\{z, w\}$ is one of the pairs $P_{i}$ and $g \in G$ then $d \rho(g)_{w} \circ \alpha_{z}=\alpha_{g z} \circ d \rho(g)_{z}$. We denote the collection of the $\alpha_{z}, z \in \mathcal{P}$, by $\alpha$.

The connected sum of $\rho$ along $(\mathcal{P}, \alpha)$ is constructed as follows. Choose a $G$-invariant auxiliary Riemannian metric on $M$. Let $r>0$ be sufficiently small so that the $2 r$-balls around all points $x_{i}, y_{i}$ are pairwise disjoint. Via the exponential map, the linear conjugacies $\alpha_{x_{i}}, \alpha_{y_{i}}$ induce a (smooth) conjugacy between the actions $G_{i} \curvearrowright B_{2 r}\left(x_{i}\right)$ and $G_{i} \curvearrowright B_{2 r}\left(y_{i}\right)$. We delete the open balls $B_{r}\left(x_{i}\right)$ and $B_{r}\left(y_{i}\right)$ and glue $G$-equivariantly along the boundary spheres. We obtain a new action $\rho_{\mathcal{P}}: G \curvearrowright M_{\mathcal{P}}$. The manifold $M_{\mathcal{P}}$ admits a natural smooth structure such that the action $\rho_{\mathcal{P}}$ is smooth. The smooth conjugacy class of $\rho_{\mathcal{P}}$ depends only on $\mathcal{P}$ and $\alpha$. (We will suppress the dependence on $\alpha$ in our notation.)

If $G$ does not act transitively on $\mathcal{P}$, one can break up the procedure into several steps: Suppose that $\mathcal{P}$ decomposes as the disjoint union $\mathcal{P}=\mathcal{P}_{1} \cup \dot{\cup} \mathcal{P}_{2}$ of $G$-invariant subfamilies $\mathcal{P}_{i}$. Then $\rho_{\mathcal{P}}: G \curvearrowright M_{\mathcal{P}}$ is smoothly conjugate to $\left(\rho_{\mathcal{P}_{1}}\right)_{\mathcal{P}_{2}}: G \curvearrowright\left(M_{\mathcal{P}_{1}}\right)_{\mathcal{P}_{2}}$, $\rho_{\mathcal{P}} \cong\left(\rho_{\mathcal{P}_{1}}\right)_{\mathcal{P}_{2}}$. 
It will be useful to consider the finite graph $\Gamma$ associated to $M$ and $\mathcal{P}$ as follows: We take a vertex for each connected component of $M$ and draw for every $i$ an edge between the vertices corresponding to the components containing $x_{i}$ and $y_{i}$. (Edges can be loops, of course.) There is a natural action $G \curvearrowright \Gamma$ induced by $\rho$.

In the following situation, the connected sum is trivial.

Lemma 2.9 (Trivial summand) Suppose that $M$ decomposes as the disjoint union $M=M_{1} \dot{\cup} M_{2}$ of $G$-invariant closed manifolds $M_{i}$, ie the $M_{i}$ are $G$-invariant unions of connected components of $M$. Assume more specifically that $M_{2}$ is a union of 3-spheres, $M_{2}=\dot{\bigcup}_{i \in I} S_{i}^{3}$, that $x_{i} \in M_{1}$ and $y_{i} \in S_{i}^{3}$, and that the action $G \curvearrowright M_{2}$ (equivalently, the actions $G_{i} \curvearrowright S_{i}^{3}$ ) are standard.

Then $\rho_{\mathcal{P}}$ is smoothly conjugate to $\left.\rho\right|_{M_{1}}$.

Proof Consider a $G$-invariant family of disjoint small balls $B_{2 r}\left(x_{i}\right) \subset M_{1}$ and $B_{2 r}\left(y_{i}\right) \subset S_{i}^{3}$ as above. The $G_{i}$-actions on $\bar{B}_{r}\left(x_{i}\right)$ and the complement of $B_{r}\left(y_{i}\right)$ in $S_{i}^{3}$ are conjugate. Thus, in forming the connected sum, we glue back in what we took out.

We will be especially interested in the situation when $M_{\mathcal{P}}$ is irreducible.

Proposition 2.10 Suppose that $M_{\mathcal{P}}$ is irreducible and connected. Suppose furthermore that the action $\rho$ is standard on the union of all components of $M$ diffeomorphic to $S^{3}$.

(i) If $M_{\mathcal{P}} \cong S^{3}$, then $\rho_{\mathcal{P}}$ is standard.

(ii) If $M_{\mathcal{P}} \not S^{3}$, then there exists a unique connected component $M_{0}$ of $M$ diffeomorphic to $M_{\mathcal{P}}$. It is preserved by $\rho$ and $\rho_{\mathcal{P}}$ is smoothly conjugate to $\left.\rho\right|_{M_{0}}$.

Proof Under our assumption, the graph $\Gamma$ is connected. Since $M_{\mathcal{P}}$ is irreducible, $\Gamma$ cannot contain cycles or loops and thus is a tree.

(ii) Since the prime decomposition of $M_{\mathcal{P}}$ is trivial, a unique such component $M_{0}$ of $M$ exists and all other components are diffeomorphic to $S^{3}$. The vertex $v_{0}$ of $\Gamma$ corresponding to $M_{0}$ is fixed by $G$.

If $\Gamma$ is just a point, the assertion is trivial. Suppose that $\Gamma$ is not a point. We choose $M_{2} \subset M$ as the union of the $S^{3}$-components which correspond to the endpoints of $\Gamma$ different from $v_{0}$. Let $M_{1}=M-M_{2}$. We have $M_{0} \subseteq M_{1}$. Each component of $M_{2}$ intersects a unique pair $P_{i}$ in exactly one point. We denote the subfamily of these $P_{i}$ by $\mathcal{P}_{\text {out }}$ and put $\mathcal{P}_{\text {inn }}=\mathcal{P}-\mathcal{P}_{\text {out }}$. According to Lemma 2.9, $\left.\rho_{\mathcal{P}_{\text {out }}} \cong \rho\right|_{M_{1}}$. Furthermore, 
$\rho_{\mathcal{P}} \cong\left(\rho_{\mathcal{P}_{\text {out }}}\right)_{\mathcal{P}_{\text {inn }}}$ and thus $\rho_{\mathcal{P}} \cong\left(\left.\rho\right|_{M_{1}}\right)_{\mathcal{P}_{\text {inn }}}$. We may replace $\rho$ by $\left.\rho\right|_{M_{1}}$ and $\mathcal{P}$ by $\mathcal{P}_{\text {inn }}$. After finitely many such reduction steps, we reach the case when $\Gamma$ is a point.

(i) If not all vertices of $\Gamma$ are endpoints, ie if $\Gamma$ is not a point or a segment, then we can perform a reduction step as in case (ii). We can therefore assume that $\Gamma$ is a point or a segment.

If $\Gamma$ is a point, there is nothing to show. Suppose that $\Gamma$ is a segment, ie $\mathcal{P}=\{P\}$. Then $M$ is the disjoint union of two spheres, $M=S_{1}^{3} \dot{\cup} S_{2}^{3}$, and $P=\left\{z_{1}, z_{2}\right\}$ with $z_{i} \in S_{i}^{3}$. Note that the points $z_{1}, z_{2}$ may be switched by $\rho$. Let $M$ be equipped with a spherical metric such that $\rho$ is isometric. Then $\alpha_{z_{1}}$ and $\alpha_{z_{2}}=\alpha_{z_{1}}^{-1}$ are the differentials of an involutive $G$-equivariant isometry $\Phi: M \rightarrow M$ switching $z_{1}$ and $z_{2}$. The action $\rho_{\mathcal{P}}$ can be obtained by restricting $\rho$ to the union of the hemispheres $B_{\pi / 2}\left(\widehat{z}_{i}\right)$ centered at the antipodes $\widehat{z}_{i} \in S_{i}^{3}$ of $z_{i}$, and gluing the boundary spheres along $\Phi$; compare the proof of Lemma 2.9. Thus $\rho_{\mathcal{P}}$ is standard also in this case.

\subsection{Balls invariant under isometric actions on the 3-sphere}

In this section we will prove the following auxiliary result which says that an action on a 3-ball is standard if it extends to a standard action on the 3-sphere.

Proposition 2.11 Suppose that $\rho: G \curvearrowright S^{3}$ is an isometric action (with respect to the standard spherical metric) and that $\bar{B}^{3} \subset S^{3}$ is a $\rho$-invariant smooth closed ball. Then the restricted action $\left.\rho\right|_{\bar{B}}$ is standard.

We denote $\Sigma:=\partial B, B_{1}:=B, B_{2}:=S^{3}-\bar{B}$.

If $\rho(g), g \in G$, has no fixed point on $\Sigma$ then, due to Brouwer's Fixed Point Theorem, it has at least one fixed point $p_{1}$ in $B_{1}$ and one fixed point $p_{2}$ in $B_{2}$. No geodesic segment $\sigma$ connecting them can be fixed pointwise, because $\sigma$ would intersect $\Sigma$ and there would be a fixed point in $\Sigma$, a contradiction. Since $\rho(g)$ is a spherical isometry, it follows that $p_{1}$ and $p_{2}$ are antipodal isolated fixed points. Thus $\rho(g)$ is the spherical suspension of the antipodal involution on $S^{2}$. In particular, $\rho(g)$ has order two and reverses orientation.

Assume now that $\rho(g)$ does have fixed points on $\Sigma$. Near $\Sigma$, Fix $(\rho(g))$ is an interval bundle over Fix $(\rho(g)) \cap \Sigma$. To see this, note that the normal geodesic through any fixed point on $\Sigma$ belongs to $\operatorname{Fix}(\rho(g))$.

If $q$ is an isolated fixed point of $\rho(g)$ on $\Sigma$ then $\left.\rho(g)\right|_{\Sigma}$ is conjugate to a finite order rotation and has precisely two isolated fixed points $q$ and $q^{\prime}$. Moreover, $\rho(g)$ is a finite 
order rotation on $S^{3}$ with the same rotation angle, and Fix $(\rho(g))$ is a great circle. We have $\operatorname{Fix}(\rho(g)) \cap \Sigma=\operatorname{Fix}\left(\left.\rho(g)\right|_{\Sigma}\right)=\left\{q, q^{\prime}\right\}$.

If $\rho(g)$ has no isolated fixed point on $\Sigma$ then, due to the classification of finite order isometries on $S^{2}$, the only remaining possibility is that $\left.\rho(g)\right|_{\Sigma}$ is (conjugate to) a reflection at a circle, $\rho(g)$ is a reflection at a $2-$ sphere and we have $\operatorname{Fix}(\rho(g)) \cap \Sigma=$ $\operatorname{Fix}\left(\left.\rho(g)\right|_{\Sigma}\right) \cong S^{1}$.

This gives:

Lemma 2.12 Let $h$ be a $\rho$-invariant auxiliary spherical metric on $\Sigma$. Then for any $g \in G, \rho(g)$ is isometrically conjugate to the spherical suspension of $\left.\rho(g)\right|_{(\Sigma, h)}$.

Note that the conjugating isometry might a priori depend on $g$.

We will see next that the action $\rho$ is determined by its restriction $\left.\rho\right|_{\Sigma}$ to $\Sigma$. Let us denote by $\tilde{\rho}$ the spherical suspension of $\left.\rho\right|_{(\Sigma, h)}$. Hence $\tilde{\rho}: G \curvearrowright S^{3}$ is an isometric action on the unit sphere and we may regard both actions as representations $\rho, \tilde{\rho}: G \rightarrow O(4)$.

\section{Lemma 2.13 The representations $\rho$ and $\tilde{\rho}$ are isomorphic.}

Proof According to Lemma 2.12, the characters of the two representations are equal. Therefore their complexifications $\rho_{\mathbb{C}}, \tilde{\rho}_{\mathbb{C}}: G \rightarrow O(4, \mathbb{C})$ are isomorphic (see eg Serre [30, Corollary 2 of Chapter 2.3]) ie there exists a $(\rho, \widetilde{\rho})$-equivariant complex linear isomorphism $A: \mathbb{C}^{4} \rightarrow \mathbb{C}^{4}, A \circ \rho_{\mathbb{C}}=\tilde{\rho}_{\mathbb{C}} \circ A$.

To deduce that already the real representations are isomorphic, we consider the composition $a:=\operatorname{Re}\left(\left.A\right|_{\mathbb{R}^{4}}\right): \mathbb{R}^{4} \rightarrow \mathbb{R}^{4}$, of $A$ with the $\tilde{\rho}$-equivariant canonical projection $\mathbb{C}^{4} \rightarrow \mathbb{R}^{4}$. It is a $(\rho, \widetilde{\rho})$-equivariant $\mathbb{R}$-linear homomorphism, $a \circ \rho=\tilde{\rho} \circ a$. We are done if $a$ is an isomorphism. We are also done if $a=0$, because then $i \cdot A: \mathbb{R}^{4} \rightarrow \mathbb{R}^{4}$ is an isomorphism. Otherwise we have a nontrivial decomposition $\mathbb{R}^{4} \cong \operatorname{ker}(a) \oplus \operatorname{im}(a)$ of the $\rho(G)$-module $\mathbb{R}^{4}$ as the direct sum of $\operatorname{ker}(a)$ and the submodule $\operatorname{im}(a)$ of the $\tilde{\rho}(G)$-module $\mathbb{R}^{4}$. Hence the representations $\rho$ and $\tilde{\rho}$ contain nontrivial isomorphic submodules. We split them off and apply the same reasoning to the complementary submodules. After finitely many steps, the assertion follows.

The isomorphism between the representations can be chosen orthogonal.

As a consequence of Lemma 2.13, the action $\rho$ has (at least a pair of antipodal) fixed points. Furthermore, for any $\rho$-fixed point $p$ the induced action $G \curvearrowright U T_{p} S^{3}$ is smoothly conjugate to $\left.\rho\right|_{\Sigma}$. We show next that there exists a pair of antipodal fixed points separated by $\Sigma$. 
Corollary 2.14 There exists a pair of antipodal $\rho$-fixed points $p_{1} \in B_{1}$ and $p_{2} \in B_{2}$.

Proof According to Lemma 2.13, $\rho$ is a suspension and hence Fix $(\rho(G))$ is a great sphere, a great circle or a pair of antipodal points.

If $\operatorname{Fix}(\rho(G)) \cong S^{2}$, then $\rho(G)$ has order two and is generated by the reflection at $\operatorname{Fix}(\rho(G))$. Our earlier discussion implies that $\Sigma$ intersects $\operatorname{Fix}(\rho(G))$ transversally in one circle $\gamma$ which divides $\operatorname{Fix}(\rho(G))$ into the disks $D_{i}:=B_{i} \cap \operatorname{Fix}(\rho(G))$. Let $\iota_{\text {Fix }(\rho(G))}$ be the antipodal involution on $\operatorname{Fix}(\rho(G))$. Since it has no fixed point, we have that $\iota_{\mathrm{Fix}(\rho(G))} D_{1} \nsubseteq D_{1}$. This implies that the open set $\iota_{\mathrm{Fix}(\rho(G))} D_{1}$ intersects $D_{2}$ and there exist antipodal points $p_{1} \in D_{1}$ and $p_{2} \in D_{2}$ as desired.

If $\operatorname{Fix}(\rho(G)) \cong S^{1}$ then there is a rotation $\rho(g) \in \rho(G)$ with $\operatorname{Fix}(\rho(g))=\operatorname{Fix}(\rho(G))$. It follows that $\Sigma$ intersects $\operatorname{Fix}(\rho(G))$ transversally in two points. As before, there exist points $p_{1}, p_{2} \in \operatorname{Fix}(\rho(G))$ as desired.

We can now assume that $\operatorname{Fix}(\rho(G))=\{p, \hat{p}\}$ is a pair of antipodal points and we must show that $\Sigma$ separates them. (Note that $\rho(G)$ cannot fix a point on $\Sigma$ because otherwise $\operatorname{dim}(\operatorname{Fix}(\rho(G))) \geq 1$. Thus $p, \hat{p} \notin \Sigma$.)

If $\rho(G)$ has order two and is generated by the involution with isolated fixed points $p$ and $\hat{p}$, then Brouwer's Fixed Point Theorem implies that each ball $B_{i}$ contains one of the fixed points, and we are done in this case.

Otherwise $\rho(G)$ contains nontrivial orientation preserving isometries. Any such element $\rho(g)$ is a rotation whose axis Fix $(\rho(g))$ is a great circle through $p$ and $\hat{p}$. If there exists $\rho\left(g^{\prime}\right) \in \rho(G)$ preserving Fix $(\rho(g))$ and such that $\left.\rho\left(g^{\prime}\right)\right|_{\operatorname{Fix}(\rho(g))}$ is a reflection at $\{p, \hat{p}\}$ then we are done because the $\left(\rho\left(g^{\prime}\right)\right.$-invariant) pair of points $\Sigma \cap \operatorname{Fix}(\rho(g))$ separates $p$ and $\hat{p}$. Let us call this situation (S).

We finish the proof by showing that (S) always occurs. Consider the induced action $d \rho_{p}: G \curvearrowright U T_{p} S^{3}$ on the unit tangent sphere in $p$ and in particular on the nonempty finite subset $F$ of fixed points of nontrivial rotations in $d \rho_{p}(G)$. We are in situation (S) if and only if some $d \rho_{p}(G)$-orbit in $F$ contains a pair of antipodes. Suppose that we are not in situation (S). Then $F$ must decompose into an even number of $\rho(G)$-orbits, in fact, into an even number of $H$-orbits for any subgroup $H \leq d \rho_{p}(G)$. (The action $d \rho_{p}$ commutes with the antipodal involution of $U T_{p} S^{3}$.) Let $G_{+} \leq d \rho_{p}(G)$ be the subgroup of orientation preserving isometries. It follows that the spherical quotient 2-orbifold $U T_{p} S^{3} / G_{+}$has an even nonzero number of cone points and hence is a sphere with two cone points, ie the spherical suspension of a circle of length $2 \pi / \mathrm{m}$, $m \geq 2$. So, $F$ is a pair of antipodes. $d \rho_{p}(G)$ cannot interchange them because we are not in situation (S). On the other hand, $d \rho_{p}(G)$ cannot fix any point on $U T_{p} S^{3}$ since the fixed point set of $\rho(G)$ on $S^{3}$ is 0 -dimensional. We obtain a contradiction and conclude that we are always in situation (S). 
In view of Lemma 2.13 and Corollary 2.14 we reformulate Proposition 2.11 as follows. By removing small invariant balls around $p_{1}$ and $p_{2}$ and replacing the metric, we convert $\rho$ into an isometric action $\rho_{1}: G \curvearrowright S^{2} \times I$ on the product of the unit 2-sphere with $I=[0,1]$ which acts trivially on $I$ (ie preserves top and bottom). We denote by $\bar{\rho}_{1}$ the projection of the $G$-action $\rho_{1}$ to $S^{2}$. We regard $\Sigma$ as a $\rho_{1}$-invariant embedded 2-sphere $\Sigma \subset S^{2} \times I$. Since the actions $\left.\rho_{1}\right|_{\Sigma}$ and $\bar{\rho}_{1}$ are conjugate, we have a $\left(\rho_{1}, \bar{\rho}_{1}\right)$-equivariant diffeomorphism $\psi: \Sigma \rightarrow S^{2}$.

Proposition 2.11 follows from:

Lemma 2.15 $\Sigma$ is $\rho_{1}$-equivariantly isotopic to a horizontal sphere $S^{2} \times t$.

Proof We know from Corollary 2.14: For any rotation $\rho(g)$ on $S^{3}$ each of the two intervals $p \times I$ and $\hat{p} \times I$ fixed by $\rho_{1}(g)$ intersects $\Sigma$ transversally in one point. For any reflection $\rho(g)$ at a 2 -sphere in $S^{3}, \bar{\rho}_{1}(g)$ acts on $S^{2}$ as the reflection at a great circle $\mu$, and $\Sigma$ intersects $\mu \times I$ transversally in one circle.

Case 1 Suppose first that $\rho(G)$ does contain reflections at 2-spheres. The mirror great circles in $S^{2}$ of the corresponding reflections in $\bar{\rho}_{1}(G)$ partition $S^{2}$ into isometric convex polygonal tiles which are either hemispheres, bigons or triangles. (Polygons with more than three vertices are ruled out by Gauß-Bonnet.) Every intersection point of mirror circles is a fixed point of a rotation $\bar{\rho}_{1}(g)$. Further fixed points of rotations $\bar{\rho}_{1}(g)$ can lie in the (in)centers of the tiles. This can occur only if the tiles are hemispheres, bigons or equilateral right-angled triangles. (No midpoint of an edge can be fixed by a rotation $\bar{\rho}_{1}(g)$ because then another mirror circle would have to run through this fixed point, contradicting the fact that it is not a vertex.)

Denote by $\Gamma \subset S^{2}$ the union of the mirror circles $\mu$ of all reflections in $\bar{\rho}_{1}(G)$. It is a $\bar{\rho}_{1}$-invariant great circle or connected geodesic graph. Note that, in the second case, when $\sigma$ is an edge of $\Gamma$ contained in the mirror $\mu$ then the circle $\Sigma \cap \mu \times I$ intersects both components of $\partial \sigma \times I$ transversally in one point and $\Sigma \cap \sigma \times I$ is a curve connecting them. It follows, in both cases, that $\Sigma$ can be $G$-equivariantly isotoped to be horizontal over a neighborhood of $\Gamma$. The tiles (components of $S^{2}-\Gamma$ ) are topologically disks, and for any tile $\tau$ the intersection $\Sigma \cap \bar{\tau} \times I$ is a disk (since it is bounded by a circle).

To do the isotopy over the 2-skeleton, let us divide out the $G$-action. We consider the spherical 2-orbifold $\mathcal{O}^{2}=S^{2} / \bar{\rho}_{1}(G)$. It has reflector boundary, its underlying topological space is the 2-disk, and possibly there is one cone point in the interior. The quotient 2-orbifold $\Sigma / \rho_{1}(G) \subset \mathcal{O} \times I$ is diffeomorphic to $\mathcal{O}$ and the embedding is horizontal over $\partial \mathcal{O}$. If $\mathcal{O}$ has no cone point in the interior then it follows with 
Alexander's Theorem (see eg Hatcher [13, Theorem 1.1]) that $\Sigma / \rho_{1}(G)$ can be made horizontal by an isotopy fixing the boundary, so the assertion of the Proposition holds in this case. If $\mathcal{O}$ has one cone point in its interior, the assertion is a consequence of the annulus case of the following standard result:

Sublemma 2.16 Let $\Sigma_{1} \cong S^{2}-\bigcup_{i=0}^{n} D_{i}, n \geq 0$, where the $D_{i}$ are open disks with disjoint closures. Suppose that $\Sigma_{2} \subset \Sigma_{1} \times[0,1]$ is a properly embedded surface, $\partial \Sigma_{2} \subset \partial \Sigma_{1} \times(0,1)$, and that $\phi: \Sigma_{2} \rightarrow \Sigma_{1}$ is a diffeomorphism which near the boundary coincides with the canonical projection onto $\Sigma_{1}$.

Then $\Sigma_{2}$ can be isotoped to be horizontal.

Proof Let $\alpha_{1}, \ldots, \alpha_{n}$ be disjoint properly embedded arcs in $\Sigma_{1}$ such that the union $\partial \Sigma_{1} \cup \alpha_{1} \cup \cdots \cup \alpha_{n}$ is connected. Then cutting $\Sigma_{1}$ along the $\alpha_{i}$ yields a disk. We may assume that $\Sigma_{2}$ intersects the strips $\alpha_{i} \times I$ transversally. Each intersection $\Sigma_{2} \cap \alpha_{i} \times I$ consists of an arc $\beta_{i}$ connecting the components of $\partial \alpha_{i} \times I$ and finitely many, possibly zero, circles. Note that $\partial \Sigma_{2} \cup \beta_{1} \cup \cdots \cup \beta_{n}$ is connected and hence cutting $\Sigma_{2}$ along the $\beta_{i}$ also yields a disk.

Suppose that $\gamma \subset \Sigma_{2} \cap \alpha_{i} \times I$ is a circle. It lies in the complement of $\bigcup_{j} \beta_{j}$ and thus bounds a disk $D \subset \Sigma_{2}$. Suppose in addition that $\gamma$ is innermost on $\Sigma_{2}$ in the sense that $D \cap\left(\bigcup_{j} \alpha_{j} \times I\right)$ is empty. Let $D^{\prime}$ be the disk bounded by $\gamma$ in $\alpha_{i} \times I$. ( $\gamma$ is not necessarily innermost in $\alpha_{i} \times I$, too, ie $D^{\prime}$ may intersect $\Sigma_{2}$ in other circles.) It follows from Alexander's Theorem (applied to the ball obtained from cutting $\Sigma_{1} \times I$ along the strips $\alpha_{i} \times I$ ) that the embedded 2-sphere $D \cup D^{\prime}$ bounds a 3-ball, and by a suitable isotopy we can reduce the number of circle components of the intersection of $\Sigma_{2}$ with $\bigcup_{j} \alpha_{j} \times I$. After finitely many steps we can achieve that $\Sigma_{2} \cap \alpha_{i} \times I=\beta_{i}$ for all $i$. After a suitable isotopy of $\Sigma_{2}$ rel $\partial \Sigma_{2}$ we may assume that the projection onto $\Sigma_{1}$ restricts to diffeomorphisms $\beta_{i} \stackrel{\cong}{\longrightarrow} \alpha_{i}$.

We now cut $\Sigma_{1} \times I$ along the strips $\alpha_{i} \times I$ and obtain a ball $\check{\Sigma}_{1} \times I$. The surface $\Sigma_{2}$ becomes a properly embedded disk $\breve{\Sigma}_{2} \subset \breve{\Sigma}_{1} \times I$. Moreover, $\partial \breve{\Sigma}_{2} \subset \partial \breve{\Sigma}_{1} \times(0,1)$ and the projection onto $\check{\Sigma}_{1}$ induces a diffeomorphism $\partial \check{\Sigma}_{2} \rightarrow \partial \check{\Sigma}_{1}$. Applying Alexander's Theorem once more, we conclude that there exists an isotopy of $\check{\Sigma}_{2}$ rel $\partial \check{\Sigma}_{2}$ which makes $\breve{\Sigma}_{2}$ transversal to the interval fibration, ie such that the projection onto $\breve{\Sigma}_{1}$ induces a diffeomorphism $\breve{\Sigma}_{2} \rightarrow \breve{\Sigma}_{1}$. The assertion follows.

We continue the proof of Lemma 2.15.

Case 2 If $\rho(G)$ is the group of order two generated by an involution of $S^{3}$ with two fixed points then the assertion follows from Livesay [16, Lemma 2]. (See also Hirsch and Smale [14].) This finishes the proof in the case when $\rho$ does not preserve orientation. 
Case 3 We are left with the case when $\rho$ preserves orientation. We consider the nonempty finite set $F \subset S^{2}$ of fixed points of nontrivial rotations in $\bar{\rho}_{1}(G)$. We recall that $\Sigma$ intersects every component of $F \times I$ transversally in one point. Let $\dot{S} \subset S^{2}$ be the compact subsurface obtained from removing a small (tubular) neighborhood around $F$. Let $\dot{\Sigma}=\Sigma \cap(\dot{S} \times I)$. As above, we divide out the $G$-action and consider the properly embedded surface $\dot{\Sigma} / \rho_{1}(G) \subset \dot{S} \times I / \rho_{1}(G)$. Its boundary is contained in $\partial \dot{S} \times(0,1) / \rho_{1}(G)$. Note that $\dot{\Sigma} / \rho_{1}(G) \cong \dot{S} / \bar{\rho}_{1}(G)$ because the actions $\left.\rho_{1}\right|_{\Sigma}$ and $\bar{\rho}_{1}$ are conjugate. These surfaces are spheres with 2 or 3 disks removed, corresponding to the fact that $\Sigma / \rho(G)$ is an oriented spherical 2-orbifold with cone points, and the number of cone points can only be 2 or 3 . We can choose orientations on $\Sigma$ and $S^{2}$ such that the canonical projection induces an orientation preserving diffeomorphism $\partial \dot{\Sigma} / \rho_{1}(G) \rightarrow \partial \dot{S} / \bar{\rho}_{1}(G)$. It extends to an orientation preserving diffeomorphism $\phi: \dot{\Sigma} / \rho_{1}(G) \rightarrow \dot{S} / \bar{\rho}_{1}(G)$. Now Sublemma 2.16 implies that $\dot{\Sigma} / \rho_{1}(G)$ can be isotoped to be horizontal. The assertion follows also in this case.

This concludes the proof of Lemma 2.15 .

\section{Tube-cap decomposition}

In this section, we recall some well-known material on Ricci flows and adapt it to our setting.

In a Ricci flow with surgery, the regions with sufficiently large positive scalar curvature are well approximated, up to scaling, by so-called $\kappa$ - and standard solutions. These local models are certain special Ricci flow solutions with time slices of nonnegative sectional curvature. Some of their properties are summarized in Section 3.2. Crucial for controlling the singularities of Ricci flow and hence also for our purposes, is their neck-cap geometry: Time slices of $\kappa$ - or standard solutions are mostly necklike, ie almost everywhere almost round cylindrical with the exception of at most two regions, so-called "caps", of bounded size (relative to the curvature scale).

The neck-cap alternative carries over to regions in a Riemannian 3-manifold which are well approximated by the local models. One infers that, globally, the region of sufficiently large positive scalar curvature in a time slice of a Ricci flow consists of tubes and caps, see Section 3.5. The tubes are formed by possibly very long chains of overlapping necks, see Section 3.3. One has very precise control of their geometry, namely they are almost cylindrical of varying width. In the time slice of a Ricci flow, the quality of approximation improves as scalar curvature increases. Hence the thinner a tube becomes, the better it is approximated by a round cylinder. The caps, on the other hand, enclose the small "islands" far apart from each other whose geometry is 
only roughly known; compare Section 3.4. Tubes and caps can be adjusted to yield an equivariant decomposition; cf Section 3.5.

\subsection{Some definitions and notation}

We call a diffeomorphism $\phi:\left(M_{1}, g_{1}\right) \rightarrow\left(M_{2}, g_{2}\right)$ of Riemannian manifolds an $\epsilon-$ isometry, $\epsilon>0$, if $\phi^{*} g_{2}$ is $\epsilon$-close, in the sense of a strict inequality, to $g_{1}$ in the $\mathcal{C}^{[1 / \epsilon]+1}$-topology. We call $\phi$ an $\epsilon$-homothety, if it becomes an $\epsilon$-isometry after suitably rescaling $g_{2}$.

We say that an action $\rho$ : $G \curvearrowright(M, g)$ is $\epsilon$-isometric, if $\rho(\gamma)$ is an $\epsilon$-isometry for all $\gamma \in G$.

Given a point $x$ with scalar curvature $S(x)>0$ in a Riemannian manifold, we define the distance from $x$ relative to its curvature scale by $\tilde{d}(x, \cdot):=S^{1 / 2}(x) \cdot d(x, \cdot)$. We then define the relative radius of a subset $A$ by $\widetilde{\operatorname{rad}}(x, A):=\sup \{\widetilde{d}(x, y): y \in A\}$, and the ball $\widetilde{B}(x, r):=\{\widetilde{d}(x, \cdot)<r\}$.

The pointed Riemannian manifold $\left(M_{1}, x_{1}, g_{1}\right)$ is said to $\epsilon$-approximate $\left(M_{2}, x_{2}, g_{2}\right)$ if $S\left(x_{1}\right)>0$ and if there exists an $\epsilon$-homothety $\phi:\left(\widetilde{B}_{1 / \epsilon}^{M_{1}}\left(x_{1}\right), S\left(x_{1}\right) g_{1}\right) \rightarrow\left(V_{2}, g_{2}\right)$ onto an open subset $V_{2}$ of $M_{2}$ with $\phi\left(x_{1}\right)=x_{2}$. We will briefly say that $\left(M_{1}, x_{1}, g_{1}\right)$ is $\epsilon$-close to $\left(M_{2}, x_{2}, g_{2}\right)$. Note that this definition is scale invariant, whereas the definition of $\epsilon$-isometry is not.

Definition 3.1 (neck) Let $\left(M^{3}, g\right)$ be a Riemannian 3-manifold. We call an open subset $\mathcal{N} \subset M$ an $\epsilon$-neck, $\epsilon>0$, if there exists an $\epsilon$-homothety

$$
\phi: S^{2}(\sqrt{2}) \times\left(-\frac{1}{\epsilon}, \frac{1}{\epsilon}\right) \rightarrow \mathcal{N}
$$

from the standard round cylinder of scalar curvature 1 and length $2 / \epsilon$ onto $\mathcal{N}$. We refer to $\phi$ as a neck chart and to a point $x \in \phi\left(S^{2}(\sqrt{2}) \times\{0\}\right)$ as a center of the $\epsilon-$ neck $\mathcal{N}$.

Note that throughout this paper, all approximations are applied to time-slices only. In particular, the necks considered here are not strong necks in the sense of Perelman [27]. We denote the open subset consisting of all centers of $\epsilon$-necks by $M_{\epsilon}^{\text {neck }}$, and its complement by $M_{\epsilon}^{\mathrm{nn}}$. We measure the necklikeness in a point $x$ by the infimum $v(x):=\inf \left\{\epsilon>0: x \in M_{\epsilon}^{\text {neck }}\right\}$. For a neck chart (3.2) one observes that $v<\epsilon /(1-|a|)$ on $\phi\left(S^{2}(\sqrt{2}) \times\{a / \epsilon\}\right)$ for $-1<a<1$. Thus $v: M \rightarrow[0, \infty)$ is a continuous function. We have that $M_{\epsilon}^{\text {neck }}=\{v<\epsilon\}$. If $v$ attains the value zero in some point $x$ then that connected component of $M$ is homothetic to the complete round standard cylinder $S^{2}(\sqrt{2}) \times \mathbb{R}$ and $v \equiv 0$ there. 
The following notion will be used to describe the nonnecklike regions near singularities of Ricci flows; compare Propositions 3.4 and 3.9 below.

Definition 3.3 $((\epsilon, d)$-cap) An incomplete Riemannian 3-manifold $C$ with strictly positive scalar curvature, which is diffeomorphic to $B^{3}$ or $\mathbb{R} P^{3}-\bar{B}^{3}$, is called an $(\epsilon, d)$-cap centered at the point $x$ if the following holds: There exists an $\epsilon$-neck $\mathcal{N} \subset C$ centered at a point $z$ with $\tilde{d}(x, z)=d$ which represents the end of $C$. Furthermore, $x \notin \mathcal{N}$ and the compact set $C-\mathcal{N}$ is contained in $\widetilde{B}(x, d)$.

Note that unlike other authors we prescribe a fixed relative diameter for caps instead of just an upper diameter bound. However, this difference is inessential because in the local models of sufficiently large diameter the nonnecklike region consists of at most two components of relative bounded diameter; cf Proposition 3.4. Thus the diameter of caps may be adapted by extending their necklike ends.

\subsection{Properties of $\kappa$-solutions and standard solutions}

$\kappa$-solutions and standard solutions serve as the local models for the regions of large positive scalar curvature in Ricci flows with surgery. Detailed information can be found in Kleiner and Lott [15, Sections 38-51 and 59-65], Morgan and Tian [21, Chapters 9 and 12] and Bamler [1, Sections 5 and 7.3]. We summarize some of their properties most relevant to us. All $\kappa$-solutions considered below will be 3 -dimensional, orientable and connected. The standard solutions are assumed to have a fixed initial metric.

Rigidity The time slices of $\kappa$ - and standard solutions have nonnegative sectional curvature. The time- $t$ slices, $t>0$, of standard solutions have strictly positive sectional curvature. If the sectional curvature of a time slice of a $\kappa$-solution is not strictly positive, then the $\kappa$-solution is a shrinking round cylinder or its orientable smooth $\mathbb{Z}_{2}$-quotient. In particular, its time slices are noncompact. (Note that a cyclic quotient of the shrinking round cylinder is not a $\kappa$-solution because time slices far back in the past are arbitrarily collapsed.)

Topological classification The topology of the time slice of a $\kappa$-solution with strictly positive sectional curvature can be derived from general results about positively curved manifolds. It is diffeomorphic to $\mathbb{R}^{3}$ in the noncompact case (see Cheeger and Gromoll [4] and Gromoll and Meyer [9]) and to a spherical space form $S^{3} / \Gamma$ in the compact case by Hamilton [12]. The time slices of standard solutions are $\cong \mathbb{R}^{3}$ by definition. 
Universal noncollapsedness There exists a universal constant $\kappa_{0}>0$ such that any $\kappa$-solution is a $\kappa_{0}$-solution unless it is a shrinking spherical space form (with large fundamental group). Standard solutions are uniformly noncollapsed, as well.

Compactness The space of pointed $\kappa_{0}$-solutions equipped with the $\mathcal{C}^{\infty}$-topology is compact modulo scaling. Also the space of pointed standard solutions (with fixed initial condition and with the tip of the time -0 slice as base point) is compact $[15$, Lemma 64.1].

Let $\left(N_{i}, x_{i}^{\prime}\right)$ be a sequence of pointed time- $t_{i}$ slices of standard solutions and suppose that $\lim _{i \rightarrow \infty} t_{i}=t_{\infty} \in[0,1]$. Then, after passing to a subsequence, the renormalized time slices $S\left(x_{i}^{\prime}\right)^{1 / 2} \cdot\left(N_{i}, x_{i}^{\prime}\right)$ converge to a time slice $\left(N_{\infty}, x_{\infty}^{\prime}\right)$ of a $\kappa_{0}$ - or a renormalized standard solution. The limit is a time slice of a $\kappa_{0}$-solution if $t_{\infty}=1$ or if $x_{i}^{\prime} \rightarrow \infty$ (on the manifold underlying the initial condition of standard solutions); cf [15, Lemmas 61.1 and 63.1]. In the latter case, the limit is the round cylinder with scalar curvature $\equiv 1$. In particular, the space of all (curvature) renormalized pointed time slices of $\kappa_{0}$-solutions or standard solutions is compact.

These compactness results yield uniform bounds for all (scale invariant) geometric quantities; compare eg Addendum 3.5 below.

Mostly necklike Time slices of $\kappa$-solutions or standard solutions are almost everywhere almost round cylindrical with the exception of at most two caps of bounded size. More precisely, one has the following information:

Proposition 3.4 (Caps in local models) For any sufficiently small $\epsilon>0$ there exist constants $D^{\prime}(\epsilon)>d^{\prime}(\epsilon)>0$ such that the following hold:

(i) Suppose that $\left(N, x^{\prime}\right)$ is a pointed time slice of a $\kappa_{0}$ - or standard solution and that $x^{\prime} \in N_{\epsilon}^{\mathrm{nn}}$ with $\widetilde{\operatorname{rad}}\left(x^{\prime}, N\right)>D^{\prime}$. Then $x^{\prime}$ is the center of an $\left(\epsilon, d^{\prime}\right)-$ cap $C \subset N$. Moreover, if $\mathcal{N} \subset C$ is an $\epsilon$-neck representing the end of $C$ as in Definition 3.3, then $\mathcal{N} \subset N_{\epsilon}^{\text {neck }}$.

(ii) If $\widehat{C} \subset N$ is another $\left(\epsilon, d^{\prime}\right)$-cap centered at a point $\hat{x}^{\prime} \notin C$, then $\widehat{C} \cap C=\varnothing$. In this case, $N$ is the time slice of a compact $\kappa_{0}$-solution and $N_{\epsilon}^{\mathrm{nn}} \subset C \cup \hat{C}$.

Regarding the geometry of the caps, the compactness theorems for the local models imply the existence of curvature and diameter bounds. (This enters already in part (ii) of the previous Proposition.) 
Addendum 3.5 (Uniform geometry of caps) There exist constants $c_{1}^{\prime}(\epsilon), c_{2}^{\prime}(\epsilon)$, $c_{3}^{\prime}\left(\epsilon, D^{\prime \prime}\right)>0$ and $\bar{d}^{\prime}(\epsilon)>0$ such that the following holds:

On the $\left(\epsilon, d^{\prime}\right)$-cap $C$ centered at $x^{\prime}$ we have $c_{1}^{\prime} S\left(x^{\prime}\right) \leq S \leq c_{2}^{\prime} S\left(x^{\prime}\right)$. Moreover $\widetilde{\operatorname{rad}}\left(x^{\prime}, C\right)<\bar{d}^{\prime}$.

If $N$ is compact with $\widetilde{\operatorname{rad}}\left(x^{\prime}, N\right)<D^{\prime \prime}$, then $\sec \geq c_{3}^{\prime} S\left(x^{\prime}\right)$ on $N$.

These facts follow from [21, Theorem 9.93], [15, Corollary 48.1, Lemma 59.7] or [1, Lemma 5.4.10, Theorems 5.4.11 and 5.4.12] for $\kappa$-solutions and from [15, Lemma 63.1] or [1, Theorem 7.3.4] for standard solutions.

\subsection{Foliating the necklike region}

Let $\left(M^{3}, g\right)$ be a Riemannian 3-manifold. In this section we discuss the global geometry of the necklike region and explain that chains of $\epsilon$-necks fit to almost cylindrical tubes, possibly long and of varying width.

In the following, $\epsilon_{0} \in(0,1 / 2008]$ will be a universal sufficiently small positive constant.

We call a unit tangent vector $v$ at a point $x \in M_{\epsilon_{0}}^{\text {neck }}$ a distant direction if there exists a geodesic segment of length $>S^{-1 / 2}(x)(1 /(2 v(x)))$ starting from $x$ in the direction $v$. The smaller $v(x)$, the closer any two distant directions $v_{1}$ and $v_{2}$ in $x$ are up to sign, $v_{1} \simeq \pm v_{2}$. In any point $x \in M_{\epsilon_{0}}^{\text {neck }}$ exists a pair of almost antipodal distant directions.

Let $x \in M_{\epsilon}^{\text {neck }}, 0<\epsilon \leq \epsilon_{0}$, and let $\phi$ be an associated neck chart; $\mathrm{cf}$ (3.2). Consider the composition $h:=\pi_{(-1 / \epsilon, 1 / \epsilon)} \circ \phi^{-1}$ where $\pi_{(-1 / \epsilon, 1 / \epsilon)}: S^{2}(\sqrt{2}) \times(-1 / \epsilon, 1 / \epsilon) \rightarrow$ $(-1 / \epsilon, 1 / \epsilon)$ denotes the projection onto the interval factor. If $\epsilon$ is sufficiently small, then the level sets $h^{-1}(t)$ are almost totally geodesic and almost round 2-spheres with scalar curvature $\simeq S(x)$. Those not too far from $x$, say with $-1 /(2 \epsilon)<t<1 /(2 \epsilon)$, are almost orthogonal to distant directions in $x$. Note that $\|d h\| \simeq S^{1 / 2}$.

Let $h_{i}(i=1,2)$ be two such functions associated to $\epsilon$-neck charts $\phi_{i}$, and let $V_{i}=\phi_{i}\left(S^{2}(\sqrt{2}) \times(-1 /(2 \epsilon), 1 /(2 \epsilon))\right)$ be the central halves of the corresponding necks $\mathcal{N}_{i}$. Note that the coordinate change $\phi_{2}^{-1} \circ \phi_{1}$ between the two neck charts is close to an isometry of the standard round cylinder (in a $\mathcal{C}^{k}$-topology for large $k$ ). In particular, after adjusting the signs of the $h_{i}$ if necessary, the 1 -forms $d h_{i}$ are close to each other on the overlap $V_{1} \cap V_{2}$, and so are the plane fields ker $d h_{i}$ and the $h_{i}$-level spheres through any point $x \in V_{1} \cap V_{2}$. The latter ones can be identified eg by following the gradient flow lines of $h_{1}$ or $h_{2}$.

To obtain the global picture, we now cover $M_{\epsilon_{0}}^{\text {neck }}$ with $\epsilon$-necks. By interpolating the associated (pairs of) 1 -forms $\pm d h$ (which may be understood as sections 
of $\left.T^{*} M /\{ \pm 1\}\right)$ we will obtain a global foliation by almost totally geodesic and almost round 2-spheres which are cross sections of $\epsilon$-necks. The geometry of the foliation approaches the standard foliation of the round cylinder by totally geodesic $2-$ spheres as the necklikeness approaches zero.

Lemma 3.6 (Foliation by 2 -spheres) There exists an open subset $F$ with $\overline{M_{\epsilon_{0}}^{\text {neck }}} \subseteq$ $F \subseteq M$, a closed smooth $1-$ form $\alpha$ on $F$ and a monotonically increasing function $\theta:\left[0, \epsilon_{0}\right) \rightarrow[0, \infty)$, continuous in 0 with $\theta(0)=0$, such that the following properties are satisfied:

- $\|\alpha\| \simeq S^{1 / 2}$, ie $1-\theta \circ v \leq S^{-1 / 2}\|\alpha\| \leq 1+\theta \circ v$.

- The complete integral manifolds of the plane field $\operatorname{ker} \alpha$ are 2 -spheres foliating $F$. We denote this foliation by $\mathcal{F}$.

- Let $x \in F$. Then, up to scaling, the foliation $\mathcal{F}$ is on $\widetilde{B}(x, 1 /(2 v(x))) \cap F$ $\theta(v(x))$-close in the $\mathcal{C}^{[1 / \theta(v(x))]+1}$-topology to the foliation of the standard round cylinder by totally geodesic cross-sectional $2-$ spheres.

Proof We exclude the trivial situation when $v$ attains the value zero and assume that $v>0$ everywhere on $M_{\epsilon_{0}}^{\text {neck }}$.

To (almost) optimize the quality of approximation, we choose for each point $x \in M_{\epsilon_{0}}^{\text {neck }}$ a constant $\epsilon(x) \in\left(0, \epsilon_{0}\right)$ with $\epsilon(x)<\frac{101}{100} v(x)$ and realize $x$ as the center of an $\epsilon(x)-$ neck $\mathcal{N}_{x}$ with neck chart $\phi_{x} ; \operatorname{cf}(3.2)$. For all these necks we consider their central halves

$$
V_{x}=\phi_{x}\left(S^{2}(\sqrt{2}) \times\left(-\frac{1}{2 \epsilon(x)}, \frac{1}{2 \epsilon(x)}\right)\right)
$$

and thirds

$$
W_{x}=\phi_{x}\left(S^{2}(\sqrt{2}) \times\left(-\frac{1}{3 \epsilon(x)}, \frac{1}{3 \epsilon(x)}\right)\right)
$$

and the exact 1 -forms $\alpha_{x}=d h_{x}$ on $V_{x}$. Using a partition of unity on $M$ subordinate to the open covering by the $V_{x}$ and the interior of $M-\bigcup_{x} W_{x}$, we interpolate the forms $\pm \alpha_{x}$ to obtain a closed 1-form $\pm \alpha$ on $\bigcup_{x} W_{x}$ (locally) well-defined up to sign, that is, a section of $T^{*} M /\{ \pm 1\}$. (More precisely, for every point $y \in \bigcup_{x} W_{x}$ we choose signs $\epsilon_{x, y} \in\{ \pm 1\}$ such that $\epsilon_{x, y} \alpha_{x} \simeq \epsilon_{x^{\prime}, y} \alpha_{x^{\prime}}$ near $y$ if $y \in V_{x} \cap V_{x^{\prime}}$, and then interpolate the forms $\epsilon_{x, y} \alpha_{x}$ near $y$.)

The plane field $\operatorname{ker}( \pm \alpha)$ on $\bigcup_{x} W_{x}$ is integrable and hence tangent to a 2-dimensional foliation $\mathcal{F}$. For any $y \in W_{x}$ the leaf $\mathcal{F}_{y}$ through $y$ is a 2 -sphere close to the 2 -sphere $h_{x}^{-1}(t)$ through $y$ (because it is a level set of a local primitive $f$ of $\alpha$, and $f$ is close to $\pm h_{x}+$ const). The approximation and the geometric properties of the leaves improve 
as $v$ decreases. We take $F$ to be a saturated open subset of $\bigcup_{x} W_{x}$ which contains all leaves of $\mathcal{F}$ meeting the closure of $M_{\epsilon_{0}}^{\text {neck }}$. We can also arrange that $\partial F$ is a disjoint union of embedded 2 -spheres.

The leaf space of $\mathcal{F}$ is a 1 -manifold. Therefore we can globally choose a sign for $\pm \alpha$, ie lift the section $\pm \alpha$ of $T^{*} M /\{ \pm 1\}$ to a section $\alpha$ of $T^{*} M$.

Suppose now that in addition we are given an isometric action $\rho: G \curvearrowright M$ of a finite group. Then the above construction can be done equivariantly.

Lemma 3.7 (Equivariant foliation) The set $F$ and its foliation $\mathcal{F}$ obtained in Lemma 3.6 can be chosen $\rho$-invariant.

Proof The family of embeddings $\phi$ and the partition of unity can be chosen $G-$ invariantly. Then the resulting section $\pm \alpha$ of $T^{*} M /\{ \pm 1\}$ is also $G$-invariant.

Given a subgroup $H \leq G$, we say that an $\epsilon$-neck $\mathcal{N} \subset M, 0<\epsilon \leq \epsilon_{0}$, is $H-$ equivariant or an $(H, \epsilon)-n e c k$, if it is $\rho(H)$-invariant as a subset and if the neck chart (3.2) can be chosen such that the pulled-back action $\phi^{*}\left(\left.\rho\right|_{H}\right)$ is isometric (with respect to the cylinder metric).

For $0<\epsilon \leq \epsilon_{0}$, we denote by $M_{H, \epsilon}^{\text {neck }}$ the subset of centers of $(H, \epsilon)-$ necks. It is contained in the union $\mathcal{F}_{H}$ of $H$-invariant leaves of the equivariant foliation $\mathcal{F}$ given by Lemma 3.7, $M_{H, \epsilon}^{\text {neck }} \subseteq \mathcal{F}_{H} \cap M_{\epsilon}^{\text {neck }}$; it is a union of $\mathcal{F}$-leaves and open in $\mathcal{F}_{H}$. We define the equivariant necklikeness $v_{H}: M_{H, \epsilon_{0}}^{\text {neck }} \rightarrow\left[0, \epsilon_{0}\right)$ analogously by $v_{H}(x):=\inf \left\{\epsilon>0: x \in M_{H, \epsilon}^{\text {neck }}\right\}$.

The next observation says that we can replace necks by equivariant ones. This becomes relevant when one wants to perform surgery on the Ricci flow equivariantly; cf Section 5.1.

Lemma 3.8 (Equivariant necks) There exists a constant $\epsilon_{0}^{G} \in\left(0, \epsilon_{0}\right]$ and a monotonically increasing function $f:\left[0, \epsilon_{0}^{G}\right] \rightarrow\left[0, \epsilon_{0}\right]$, continuous in 0 with $f(0)=0$, such that for any subgroup $H \leq G$ holds $v_{H} \leq f \circ v$ on $M_{\epsilon_{0}^{G}}^{\text {neck }} \cap \mathcal{F}_{H} \subseteq M_{H, \epsilon_{0}}^{\text {neck }}$.

Proof Let $x \in M_{\epsilon_{0}}^{\text {neck }} \cap \mathcal{F}_{H}$ and let us normalize so that $S(x)=1$. As before, we denote by $\mathcal{F}_{x}$ the leaf of $\mathcal{F}$ through $x$. If $v(x)$ is small, the metric is on a ball of radius $\simeq 1 /(2 v(x))$ around $x$ very close (in a topology of large smoothness degree) to the standard round cylinder. Furthermore, the foliation $\mathcal{F}$ is on this ball very close to the foliation of the standard round cylinder by totally geodesic cross-sectional 2-spheres. It follows that the $\rho(H)$-invariant metric $\left.g\right|_{\mathcal{F}_{x}}$ is close, in terms of $\nu(x)$ and $|G|$, to a 
$\rho(H)$-invariant round metric with scalar curvature $\equiv 1$. Let $\phi_{0}: S^{2}(\sqrt{2}) \rightarrow \mathcal{F}_{x}$ be a corresponding almost isometric diffeomorphism which is $\left(\hat{\rho}_{0},\left.\rho\right|_{H}\right)$-equivariant with respect to a suitable isometric action $\hat{\rho}_{0}: H \curvearrowright S^{2}(\sqrt{2})$. Using the $\rho$-invariant line field perpendicular to $\mathcal{F}$ and its integral lines, we can extend $\phi_{0}$ to a $\left(\hat{\rho},\left.\rho\right|_{H}\right)$-equivariant embedding

$$
\phi: S^{2}(\sqrt{2}) \times\left(-\frac{1}{2 v(x)}, \frac{1}{2 v(x)}\right) \hookrightarrow M,\left.\quad \phi\right|_{S^{2}(\sqrt{2}) \times\{0\}}=\phi_{0},
$$

where $\hat{\rho}$ is a suitable extension of $\hat{\rho}_{0}$ to an isometric action of $H$ on

$$
S^{2}(\sqrt{2}) \times\left(-\frac{1}{2 v(x)}, \frac{1}{2 v(x)}\right) .
$$

Given $\epsilon>0$, the restriction of $\phi$ to $S^{2}(\sqrt{2}) \times\left(-\frac{1}{\epsilon}, \frac{1}{\epsilon}\right)$ is arbitrarily close to an isometry provided that $v(x)$ is sufficiently small.

\subsection{Neck-cap geometry}

Let $\left(M^{3}, g\right)$ be a connected orientable closed Riemannian 3-manifold.

Let $\epsilon \gg \epsilon_{1}>0$. Let $A_{0}\left(\epsilon_{1}\right) \subseteq M$ be the open subset of points $x$ such that $(M, x, g)$ is $\epsilon_{1}$-approximated by a $\kappa$-solution or a standard solution $\left(N, x^{\prime}, h\right)$. Define $A_{1}\left(\epsilon, \epsilon_{1}\right):=A_{0}\left(\epsilon_{1}\right) \cap\{\widetilde{\operatorname{rad}}(\cdot, M)>D(\epsilon)\}$, with $D(\epsilon)$ to be specified in Proposition 3.9 below. Note that for $x \in A_{1}$, a $\kappa$-solution $\epsilon_{1}$-approximating $(M, x, g)$ is a $\kappa_{0}$-solution.

Suppose that $x \in A_{0}$. If $\epsilon_{1}$ is sufficiently small (in terms of $\epsilon$ ), then centers of $(\epsilon / 2)$-necks in $N$ correspond via the approximation to centers of $\epsilon$-necks in $M$. In particular, if $x^{\prime} \in N_{\epsilon / 2}^{\text {neck }}$ then $x \in M_{\epsilon}^{\text {neck }}$, respectively, if $x \in M_{\epsilon}^{\mathrm{nn}}$ then $x^{\prime} \in N_{\epsilon / 2}^{\mathrm{nn}}$.

The neck-cap alternative carries over from the local models to regions which are well approximated by them. We obtain from Proposition 3.4:

Proposition 3.9 (Caps) For any sufficiently small $\epsilon>0$ there exist constants $D(\epsilon) \gg$ $\bar{d}(\epsilon)>d(\epsilon)>0$ and $0<\epsilon_{1}^{(1)}(\epsilon) \leq \frac{1}{2 D}$ such that the following hold:

(i) If $0<\epsilon_{1} \leq \epsilon_{1}^{(1)}$ and $x \in M_{\epsilon}^{\mathrm{nn}} \cap A_{1}$, then there exists an $(\epsilon, d)$-cap $C$ centered at $x$. It satisfies $\underset{\operatorname{rad}}{ }(x, C)<\bar{d}$. Moreover, if $\mathcal{N} \subset C$ is an $\epsilon-$ neck representing the end of $C$ as in Definition 3.3, then $\mathcal{N} \subset M_{\epsilon}^{\text {neck }}$.

(ii) If $\widehat{C} \subset M$ is another $(\epsilon, d)$-cap centered at a point $\widehat{x} \in\left(M_{\epsilon}^{\mathrm{nn}} \cap A_{1}\right)-C$, then $\widehat{C} \cap C=\varnothing$.

We will refer to an $(\epsilon, d(\epsilon))$-cap in $M$ simply as an $\epsilon$-cap. 
Corollary 3.10 If $C_{1}, C_{2}$ are $\epsilon$-caps centered at $x_{1}, x_{2} \in M_{\epsilon}^{\mathrm{nn}} \cap A_{1}$, then

$$
C_{1} \cap C_{2} \neq \varnothing \Leftrightarrow C_{1} \cap M_{\epsilon}^{\mathrm{nn}} \cap A_{1}=C_{2} \cap M_{\epsilon}^{\mathrm{nn}} \cap A_{1} .
$$

Proof Direction " $\Leftarrow$ " is trivial. We prove direction " $\Rightarrow$ ". By part (ii) of the proposition, we have that $x_{2} \in C_{1}$ and $x_{1} \in C_{2}$. Let $x \in C_{1} \cap M_{\epsilon}^{\mathrm{nn}} \cap A_{1}$ and let $C$ be an $\epsilon-$ cap centered at $x$. Then $x_{1} \in C$. Hence $x_{1} \in C \cap C_{2} \neq \varnothing$ and therefore also $x \in C_{2}$.

Consequently, for $\epsilon$-caps centered at points in $M_{\epsilon}^{\mathrm{nn}} \cap A_{1}$, the relation defined by $C_{1} \sim{ }^{\prime} C_{2}$ if and only if $C_{1} \cap C_{2} \neq \varnothing$ is an equivalence relation. Equivalent caps differ only outside $M_{\epsilon}^{\mathrm{nn}} \cap A_{1}$, and inequivalent caps are disjoint. Furthermore, the relation on $M_{\epsilon}^{\mathrm{nn}} \cap A_{1}$ defined by $x_{1} \sim x_{2}$, if and only if there exists an $\epsilon$-cap $C$ containing $x_{1}$ and $x_{2}$, is an equivalence relation. The equivalence class of a point $x$ is given by $C \cap M_{\epsilon}^{\mathrm{nn}} \cap A_{1}$ for any $\epsilon$-cap containing $x$.

Note that there exists $\rho>0$ with the property that every $\epsilon$-cap $C$ centered at $x \in$ $M_{\epsilon}^{\mathrm{nn}} \cap A_{1}$ contains $\widetilde{B}(x, \rho)$. Consequently, since $M$ is closed, there can only be finitely many equivalence classes of $\epsilon$-caps.

Note that the situation when $A_{1} \subsetneq A_{0}$ is very special. There exists $x \in A_{0}$ with $\widetilde{\operatorname{rad}}(x, M) \leq D$ and hence $M$ is globally $\epsilon_{1}$-approximated by a compact $\kappa$-solution, ie by a compact $\kappa_{0}$-solution or a spherical space form. We choose the constant $\epsilon_{1}^{(1)}$ in Proposition 3.9 sufficiently small, such that in addition one has a uniform positive lower bound for sectional curvature, $\sec \geq c S(x)$ on $M$ with a constant $c(D(\epsilon))=c(\epsilon)>0$; cf the last assertion of Addendum 3.5.

\subsection{Equivariant tube-cap decomposition}

We now combine the discussions in Section 3.3 and the previous section to describe, in the equivariant case, the global geometry of the region which is well approximated by the local models. This comprises the region of sufficiently large positive scalar curvature in the time slice of a Ricci flow.

Let $\left(M^{3}, g\right)$ be again a connected orientable closed Riemannian 3-manifold and let $\rho: G \curvearrowright M$ be an isometric action by a finite group. In the following, $\epsilon>0$ denotes a sufficiently small positive constant. It determines via Proposition 3.9 the even much smaller positive constant $\epsilon_{1}$. All $\epsilon$-caps are assumed to be centered at points in $M_{\epsilon}^{\mathrm{nn}} \cap A_{1}\left(\epsilon, \epsilon_{1}\right)$. Furthermore, we suppose that $A_{1}=A_{0}$; compare the remark in the end of Section 3.4.

Let $C \subset M$ be an $\epsilon$-cap. By the construction of caps, the end of $C$ is contained in $M_{\epsilon}^{\text {neck }}$ and hence in the foliated region $F$; cf Section 3.3. Let $T$ be the connected 
component of $F$ containing the end of $C$. We will refer to $T$ as the $\epsilon$-tube associated to $C$. Of course, $C \not \subset F$, eg for topological reasons. Thus $\partial T$ consists of two embedded 2-spheres. One boundary sphere $\partial_{\text {inn }} T$ of $T$ is contained in $C \cap M_{\epsilon}^{\mathrm{nn}}$, and the other boundary sphere $\partial_{\text {out }} T$ is contained in $M_{\epsilon}^{\mathrm{nn}}-C$. (Note that $\partial F \subset M_{\epsilon}^{\mathrm{nn}}$.)

We consider now two situations which are of special interest to us.

Situation $1 \quad\left(M=A_{1}.\right)$ This will cover the case of extinction to be discussed in Section 5.2.

If $M_{\epsilon}^{\text {neck }}=M$, then $M$ is globally foliated and consequently $M \cong S^{2} \times S^{1}$.

If $M_{\epsilon}^{\text {neck }} \subsetneq M$, let $C \subset M$ be an $\epsilon$-cap and let $T$ be the $\epsilon$-tube associated to $C$. The boundary sphere $\partial_{\text {out }} T$ is contained in a different $\epsilon$-cap $\widehat{C}$. The caps $C$ and $\widehat{C}$ are disjoint, and we obtain the tube-cap decomposition

$$
M=C \cup T \cup \widehat{C}
$$

of $M$. In this case, there are exactly two equivalence classes of $\epsilon$-caps.

Situation 2 This more general situation which we describe now is tailored to apply to the highly curved region in a Ricci flow short before a surgery time; cf Section 5.3.

By a funnel $Y \subset M_{\epsilon}^{\text {neck }}$ we mean a submanifold $\cong S^{2} \times[0,1]$ which is a union of leaves of the foliation $\mathcal{F}$, and which has one highly curved boundary sphere $\partial_{h} Y$ and one boundary sphere $\partial_{l} Y$ with lower curvature. Quantitatively, we require that $\left.\min S\right|_{\partial_{h} Y}>\left.C \cdot \max S\right|_{\partial_{l} Y}$, where $C(\epsilon) \gg 1$ is a constant greater than the bound for the possible oscillation of scalar curvature on $\epsilon$-caps. That is, $C$ is chosen as follows (cf Addendum 3.5): If $y_{1}$ and $y_{2}$ are points in any $\epsilon$-cap then $S\left(y_{1}\right)<C \cdot S\left(y_{2}\right)$.

Suppose that we are given a finite $\rho$-invariant family of pairwise disjoint funnels $Y_{j} \subset M_{\epsilon}^{\text {neck }}$ (corresponding later to parts of horns), that $M_{1} \subset M$ is a union of components of $M-\bigcup_{j} Y_{j}$ such that $\partial M_{1}=\bigcup_{j} \partial_{h} Y_{j}$, and furthermore that $M_{1} \subset A_{1}$.

We restrict our attention to those $\epsilon$-caps which intersect $M_{1}$. Under our assumptions, every such cap $C$ is contained in $M_{1} \cup Y, Y:=\bigcup_{j} Y_{j}$, and all $\epsilon$-caps equivalent to $C$ also intersect $M_{1}$.

Let $C_{1}, \ldots, C_{m}$ be representatives for the equivalence classes of these caps, $m \geq 0$. We have that $C_{i} \cap M_{\epsilon}^{\mathrm{nn}} \subset A_{1}$. Let $T_{i}^{\prime}$ be the $\epsilon$-tube associated to $C_{i}$. It is the unique component of $F$ such that every $\epsilon$-cap equivalent to $C_{i}$ contains precisely one of its boundary spheres, namely $\partial_{\mathrm{inn}} T_{i}^{\prime}:=\partial T_{i}^{\prime} \cap C_{i}$. In particular, $T_{i}^{\prime}$ depends only on the equivalence class $\left[C_{i}\right]$. 
If $T_{i}^{\prime} \cap Y \neq \varnothing$, then we truncate $T_{i}^{\prime}$ where it leaves $\overline{M_{1}}$. That is, we replace $T_{i}^{\prime}$ by the compact subtube $T_{i} \subseteq T_{i}^{\prime}$ with the properties that $\partial_{\mathrm{inn}} T_{i}=\partial_{\mathrm{inn}} T_{i}^{\prime}$ and $\partial_{\text {out }} T_{i}=T_{i} \cap Y$ is a sphere component of $\partial_{h} Y$. Otherwise, we put $T_{i}:=T_{i}^{\prime}$.

If $T_{i} \cap \partial Y=\varnothing$, then $T_{i} \subset M_{1}$ and $\partial_{\text {out }} T_{i}$ is contained in a different cap $C_{\iota(i)}, \iota(i) \neq i$. As in Situation 1 above,

$$
C_{i} \cup T_{i} \cup C_{l(i)}
$$

is a closed connected component of $M_{1}$. On the other hand, if $T_{i} \cap \partial Y \neq \varnothing$ and hence $T_{i} \cap \partial Y=\partial_{\text {out }} T_{i}$, then

$$
C_{i} \cup T_{i}
$$

is a component of $\overline{M_{1}}$ with one boundary sphere. We call it an $\epsilon$-tentacle. All caps $C_{i}$ occur in a component (3.12) or (3.13).

There may be further components of $M_{1}$ that are contained in $F$. They are either closed and $\cong S^{2} \times S^{1}$, or they are tubes $\cong S^{2} \times[0,1]$ whose boundary spheres are components of $\partial_{h} Y$.

This provides the tube-cap decomposition of $M_{1}$.

Equivariance In Situation 2, the tube $T_{i}$ depends only on the equivalence class $\left[C_{i}\right]$. Therefore the subgroup $\operatorname{Stab}_{G}\left(\left[C_{i}\right]\right)$ of $G$ preserves $T_{i}$, every $\mathcal{F}$-leaf contained in $T_{i}$ and hence also the cap $C_{i}$. The union of tubes $T_{i}$ is invariant under the whole group $G$, and the caps $C_{i}$ can be adjusted such that their union is $\rho$-invariant, too. Situation 1 is analogous to the case (3.12) of Situation 2. Thus the tube-neck decomposition can also always be done equivariantly.

\section{Actions on caps}

In order to compare the $G$-actions before and after surgery, see Section 5.3 below, one needs to classify the action on the highly curved region near the singularity of the Ricci flow, ie short before the surgery. On the necklike part, one has very precise control on the geometry and hence also on the action. On the other hand, the caps are diffeomorphic to $B^{3}$ or $\mathbb{R} P^{3}-\bar{B}^{3}$ but at least in the first case there is relatively little information about their geometry. However, caps may have nontrivial stabilizers in $G$ and one must verify that their actions on the caps are standard. This is the aim of the present chapter. For an alternative way of controlling the action on caps (which does not require Proposition 2.11) we refer to [7]. 
Since we are working with nonequivariant approximations by local models, we obtain almost isometric actions on local models which are not defined everywhere but only on a large region whose size depends on the quality of the approximation. The key step, see Section 4.1, is to approximate such partially defined almost isometric actions by globally defined isometric actions on nearby local models with symmetries. This is possible due to the compactness properties of the spaces of local models. We then verify in Section 4.2 that isometric actions on local models are standard, as well as their restrictions to invariant caps. From this we deduce in Section 4.3 that the actions on caps are indeed standard.

\subsection{Approximating almost isometric actions on local models by isometric ones}

Suppose that $\rho: G \curvearrowright(M, g)$ is an isometric action, that $(N, h)$ is a local model with normalized curvature in a base point, $S\left(x^{\prime}\right)=1$, and that $\phi: \widetilde{B}^{N}\left(x^{\prime}, 1 / \epsilon_{1}\right) \rightarrow M$ is an $\epsilon_{1}$-homothetic embedding whose image contains an open subset $V$ preserved by a subgroup $H \leq G$. Then the pulled-back action $\left.\phi^{*} \rho\right|_{H}: H \curvearrowright \phi^{-1}(V)$ is $\tilde{\epsilon}_{1}\left(\epsilon_{1}\right)-$ isometric with $\tilde{\epsilon}_{1}\left(\epsilon_{1}\right) \rightarrow 0$ for $\epsilon_{1} \rightarrow 0$; compare the definitions in Section 3.1.

The next result allows to improve approximations by almost isometric partial actions to approximations by global isometric actions.

Lemma 4.1 For $a, \zeta>0$ and a finite group $H$ there exists $\eta(a, \zeta, H)>0$ such that: Suppose that $\left(N, x^{\prime}\right)$ is a time slice of a $\kappa_{0}$ - or a rescaled standard solution, normalized so that $S\left(x^{\prime}\right)=1$. Furthermore, for some $\epsilon_{1} \in(0, \eta]$ let $\rho: H \curvearrowright V$ be an $\epsilon_{1}$-isometric action on an open subset $V$ with $\widetilde{B}\left(x^{\prime}, \frac{99}{100}\left(1 / \epsilon_{1}\right)\right) \subseteq V \subseteq N$, and suppose that there is a $\rho$-invariant open subset $A, x^{\prime} \in A \subset V$, with $\widetilde{\operatorname{rad}}\left(x^{\prime}, A\right)<a$.

Then there exists a time slice $\left(\hat{N}, \hat{x}^{\prime}\right)$ of a $\kappa_{0}$ - or rescaled standard solution which is $\zeta$-close to $\left(N, x^{\prime}\right)$, a globally defined isometric action $\hat{\rho}: H \curvearrowright \widehat{N}$ and a $(\rho, \hat{\rho})-$ equivariant smooth embedding $\iota$ : $A \hookrightarrow \widehat{N}$.

Proof We argue by contradiction. We assume that for some $a, \zeta, H$ there exists no such $\eta$. Then there exist sequences of positive numbers $\epsilon_{1 i} \searrow 0$, of time slices of $\kappa_{0}-$ or standard solutions $\left(N_{i}, x_{i}^{\prime}\right)$ with $S\left(x_{i}^{\prime}\right)=1$, of $\epsilon_{1 i}$-isometric actions $\rho_{i}: H \curvearrowright V_{i}$ on open subsets $V_{i}, \widetilde{B}\left(x_{i}^{\prime}, \frac{99}{100}\left(1 / \epsilon_{1 i}\right)\right) \subseteq V_{i} \subseteq N_{i}$, and of $\rho_{i}(H)$-invariant open neighborhoods $A_{i}$ of $x_{i}^{\prime}$ with $\widetilde{\operatorname{rad}}\left(x_{i}^{\prime}, A_{i}\right)<a$, which violate the conclusion of the lemma for all $i$.

According to the compactness theorems for $\kappa$ - and standard solutions (cf Section 3.2) after passing to a subsequence, the $\left(N_{i}, x_{i}^{\prime}\right)$ converge smoothly to a time slice 
$\left(N_{\infty}, x_{\infty}^{\prime}\right)$ of a $\kappa_{0}$ - or a renormalized standard solution. Hence for $i$ sufficiently large, $\left(N_{i}, x_{i}^{\prime}\right)$ is $\zeta$-close to $\left(N_{\infty}, x_{\infty}^{\prime}\right)$.

The convergence of the actions follows: By the definition of closeness, given $v>0$, we have for $i \geq i(v)$ an $v$-isometric embedding $\psi_{i}$ : $\left(\widetilde{B}\left(x_{\infty}^{\prime}, \frac{1}{v}\right), x_{\infty}^{\prime}\right) \hookrightarrow\left(N_{i}, x_{i}^{\prime}\right)$ such that $\operatorname{im}\left(\psi_{i}\right) \subset V_{i}$. Our assumption implies $\widetilde{\operatorname{rad}}\left(x_{i}^{\prime}, \rho_{i}(H) x_{i}^{\prime}\right)<a$ and that there exists an open $\rho_{i}(H)$-invariant subset $U_{i}$ with $\widetilde{B}\left(x_{i}^{\prime}, \frac{1}{v}-\frac{101}{100} a\right) \subset U_{i} \subset \operatorname{im}\left(\psi_{i}\right)$. We pull the $\epsilon_{1 i}$-isometric action $\left.\rho_{i}\right|_{U_{i}}$ back to an $\tilde{\epsilon}_{i}\left(\epsilon_{1 i}, v\right)$-isometric action $\psi_{i}^{*} \rho_{i}: H \curvearrowright \psi_{i}^{-1}\left(U_{i}\right)$. Let $\left(v_{i}\right)_{i \geq i_{0}}$ be a sequence of positive numbers, $v_{i} \searrow 0$, such that $i\left(v_{i}\right) \leq i$. Then the action $\psi_{i}^{*} \rho_{i}$ is in fact $\tilde{\epsilon}_{i}\left(\epsilon_{1 i}, v_{i}\right)$-isometric, and $\tilde{\epsilon}_{i}\left(\epsilon_{1 i}, v_{i}\right) \rightarrow 0$. We have that $U_{i} \nearrow N_{\infty}$ and $\lim \sup _{i \rightarrow \infty} \widetilde{\operatorname{rad}}\left(x_{\infty}^{\prime}, \psi_{i}^{*} \rho_{i}(H) x_{\infty}^{\prime}\right) \leq a$. This implies that, after passing to a subsequence, the actions $\psi_{i}^{*} \rho_{i}$ converge to an isometric limit action $\rho_{\infty}: H \curvearrowright N_{\infty}$ with $\widetilde{\operatorname{rad}}\left(x_{\infty}^{\prime}, \rho_{\infty}(H) x_{\infty}^{\prime}\right) \leq a$.

Consider now the open subsets $\psi_{i}^{-1}\left(A_{i}\right) \subset N_{\infty}$. Their diameters are uniformly bounded, $\widetilde{\operatorname{rad}}\left(x_{\infty}^{\prime}, \psi_{i}^{-1}\left(A_{i}\right)\right)<\frac{101}{100} a$. For $i \rightarrow \infty$, the almost isometric action $\psi_{i}^{*} \rho_{i}$ and the isometric action $\rho_{\infty}$ become arbitrarily close on $\psi_{i}^{-1}\left(A_{i}\right)$ (actually on a much larger subset). Following Palais [24] and Grove and Karcher [10], we construct for large $i$ smooth maps conjugating $\left.\psi_{i}^{*} \rho_{i}\right|_{\psi_{i}^{-1}\left(A_{i}\right)}$ into $\rho_{\infty}$. For $h \in H$ the smooth maps

$$
\phi_{i, h}:=\rho_{\infty}(h)^{-1} \circ\left(\psi_{i}^{*} \rho_{i}\right)(h): \widetilde{B}\left(x_{\infty}^{\prime}, \frac{101}{100} a\right) \rightarrow N_{\infty}
$$

converge to the identity. For $i$ sufficiently large, the sets $\left\{\phi_{i, h}(x): h \in H\right\}$, where $x \in B\left(x_{\infty}^{\prime}, \frac{101}{100} a\right)$, have sufficiently small diameter so that their center of mass $c_{i}(x)$ is well-defined; see Grove and Karcher [10, Proposition 3.1]. The maps $c_{i}$ are smooth (cf [10, Proposition 3.7]) and $c_{i} \rightarrow \mathrm{id}_{N_{\infty}}$. Note that the center of the set

$$
\left\{\left(\rho_{\infty}\left(h^{\prime}\right)^{-1} \circ\left(\psi_{i}^{*} \rho_{i}\right)\left(h^{\prime}\right) \circ\left(\psi_{i}^{*} \rho_{i}\right)(h)\right)(x): h^{\prime} \in H\right\}
$$

equals $c_{i}\left(\left(\psi_{i}^{*} \rho_{i}\right)(h)(x)\right)$. On the other hand, it is the $\rho_{\infty}(h)$-image of the center of the set

$$
\left\{\left(\rho_{\infty}\left(h^{\prime} h\right)^{-1} \circ\left(\psi_{i}^{*} \rho_{i}\right)\left(h^{\prime} h\right)\right)(x): h^{\prime} \in H\right\},
$$

and the latter equals $c_{i}(x)$. So $\rho_{\infty}(h) \circ c_{i}=c_{i} \circ\left(\psi_{i}^{*} \rho_{i}\right)(h)$ on $B\left(x_{\infty}^{\prime}, \frac{101}{100} a\right)$ and thus $\rho_{\infty}(h) \circ\left(c_{i} \circ \psi_{i}^{-1}\right)=\left(c_{i} \circ \psi_{i}^{-1}\right) \circ \rho_{i}(h)$ on $A_{i}$. The existence of the $\left(\rho_{i}, \rho_{\infty}\right)-$ equivariant smooth embeddings $\iota_{i}=c_{i} \circ \psi_{i}^{-1}: A_{i} \hookrightarrow N_{\infty}$ shows that the conclusion of the lemma is satisfied for large $i$. Putting $\left(\widehat{N}, \hat{x}^{\prime}\right)=\left(N_{\infty}, x_{\infty}^{\prime}\right)$ and $\hat{\rho}=\rho_{\infty}$, we obtain a contradiction.

\subsection{Isometric actions on local models are standard}

Let $(N, h)$ be a local model, ie a time slice of a $\kappa$ - or standard solution, and let $\rho: H \curvearrowright N$ be an isometric action by a finite group. 
If $N$ is the time slice of a standard solution, it has rotational symmetry, $\operatorname{Isom}(N, h) \cong$ $O(3)$. The natural action $\operatorname{Isom}(N, h) \curvearrowright N$ is smoothly conjugate to the orthogonal action $O(3) \curvearrowright B^{3}$, and in particular the action $\rho$ is standard.

If $N$ is a round cylinder or its orientable smooth $\mathbb{Z}_{2}$-quotient, then $\rho$ is also clearly standard.

Otherwise, $N$ is the time slice of a $\kappa$-solution and has strictly positive sectional curvature. This case is covered by the following result.

Proposition 4.2 An isometric action by a finite group on a complete 3-manifold with strictly positive sectional curvature is smoothly conjugate to an

(i) orthogonal action on $\mathbb{R}^{3}$ if the manifold is noncompact;

(ii) isometric action on a spherical space form if the manifold is compact.

The compact case is a direct consequence of Hamilton [12]. The noncompact case follows from an equivariant version of the Soul Theorem which holds in all dimensions:

Proposition 4.3 Let $W^{n}$ be a complete noncompact Riemannian manifold with strictly positive sectional curvature. Suppose that $H$ is a finite group and that $\rho: H \curvearrowright W$ is an isometric action. Then $H$ fixes a point and $\rho$ is smoothly conjugate to an orthogonal action on $\mathbb{R}^{n}$.

Proof One follows the usual proof of the Soul Theorem by Gromoll and Meyer [9] and Cheeger and Gromoll [4] (see eg the nice presentation in Meyer [19, Chapters 3.2 and 3.6]) in the special case of strictly positive curvature and makes all constructions group invariant.

In a bit more detail: Starting from the collection of all geodesic rays with initial points in a fixed $H$-orbit, one constructs an exhaustion $\left(C_{t}\right)_{t \geq 0}$ of $W$ by $H$-invariant compact totally convex subsets. We may assume that $C_{0}$ has nonempty interior. Since the sectional curvature is strictly positive, $C_{0}$ contains a unique point $s$ at maximal distance from its boundary. It is fixed by $H$ and it is a soul for $M$. The distance function $d(s, \cdot)$ has no critical points (in the sense of Grove and Shiohama [11]) besides $s$. One can construct an $H$-invariant gradient-like vector field $X$ for $d(s, \cdot)$ on $W-\{s\}$. It can be arranged that $X$ coincides with the radial vector field $\nabla d(s, \cdot)$ near $s$. Using the flow of $X$ one obtains a smooth conjugacy between $\rho$ and its induced orthogonal action $d \rho_{s}$ on $T_{s} W \cong \mathbb{R}^{n}$. Near $s$ it is given by the (inverse of the) exponential map. $\square$ 
Remark 4.4 In the compact case, if the local model $N$ has large diameter, then the possibilities for the actions are more restricted, as the discussion in Section 3.5 shows. There is a $\rho$-invariant tube-cap decomposition $N=C_{1} \cup T \cup C_{2}$ and the central leaf $\Sigma$ of the tube $T$ is preserved by $\rho$.

We now apply Proposition 2.11 to deduce that for any cap in a local model, which is invariant under an isometric action, the restricted action on the cap is standard.

Corollary 4.5 Suppose that $\bar{C} \subset N$ is a compact $\rho$-invariant submanifold diffeomorphic to $\bar{B}^{3}$ or $\mathbb{R} P^{3}-B^{3}$. Then the restricted action $\rho \mid \bar{C}$ is standard.

Proof When $N$ is noncompact, it can be compactified by adding one or two points to a smooth manifold $\cong S^{3}$ or $\mathbb{R} P^{3}$, and the action $\rho$ can be extended to a smooth standard action. The latter is clear when $N$ is the time slice of a standard solution or when $N$ is isometric to $S^{2} \times \mathbb{R}$ or $S^{2} \times \mathbb{Z}_{2} \mathbb{R}$. It follows from Proposition 4.3 when $N$ has strictly positive sectional curvature. In view of Proposition 4.2 (ii), we may therefore assume that $N$ is metrically a spherical space form $S^{3} / \Gamma$.

Suppose first that $\bar{C}$ is a ball. Then $\bar{C}$ can be lifted to a closed ball $\bar{B} \subset S^{3}$ and $\rho$ can be lifted to an isometric action $\tilde{\rho}$ : $H \curvearrowright S^{3}$ preserving $\bar{B}$. Now Proposition 2.11 implies that the restricted action $\left.\widetilde{\rho}\right|_{\bar{B}}$ is standard, and therefore also $\rho \mid \bar{C}$.

We are left with the case when $\bar{C} \cong \mathbb{R} P^{3}-B^{3}$. Since $S^{3} / \Gamma$ is irreducible, the 2 -sphere $\partial C$ bounds on the other side a ball $B^{\prime}$, and hence $N \cong \mathbb{R} P^{3}$. As before, Proposition 2.11 implies that $\left.\rho\right|_{\bar{B}^{\prime}}$ is standard. It follows that $\partial B^{\prime}=\partial C$ can be $\rho$-equivariantly isotoped to a (small) round sphere, and that also the action $\rho \mid \bar{C}$ is standard.

\subsection{Actions on caps are standard}

We take up the discussion of the equivariant tube-cap decomposition from Section 3.5. Let $x \in M_{\epsilon}^{\mathrm{nn}} \cap A_{1}$ and let $C \subset M$ be an $\epsilon$-cap centered at $x$ as given by Proposition 3.9. Every other $\epsilon$-cap that intersects $C$ agrees with $C$ on $M_{\epsilon}^{\mathrm{nn}} \cap A_{1}$; cf Corollary 3.10. Let $H:=\operatorname{Stab}_{G}\left(C \cap M_{\epsilon}^{\mathrm{nn}} \cap A_{1}\right)$. Then $C$ can be modified to be $H$-invariant, and we have that $\gamma C \cap C=\varnothing$ for all $\gamma \in G-H$.

Proposition 4.6 There exists $0<\epsilon_{1}^{(2)}(H, \epsilon) \leq \epsilon_{1}^{(1)}$ such that for $0<\epsilon_{1} \leq \epsilon_{1}^{(2)}$ holds: Let $x \in M_{\epsilon}^{\text {neck }} \cap A_{1}$ be center of an $H$-invariant $\epsilon$-cap $C, H=\operatorname{Stab}_{G}\left(C \cap M_{\epsilon}^{\mathrm{nn}} \cap A_{1}\right)$, then the restricted action $\rho \mid \bar{C}: H \curvearrowright \bar{C}$ is standard. 
Proof Let $\left(N, x^{\prime}, h\right)$ be a time slice of a $\kappa_{0}-$ or rescaled standard solution, normalized so that $S\left(x^{\prime}\right)=1$. Suppose that $\left(N, x^{\prime}, h\right) \epsilon_{1}$-approximates $(M, x, g)$, and let $\phi: \widetilde{B}\left(x^{\prime}, 1 / \epsilon_{1}\right) \hookrightarrow M$ be an $\epsilon_{1}$-homothetic embedding realizing the approximation.

Since $\widetilde{\operatorname{rad}}(x, \rho(H) x) \leq \widetilde{\operatorname{rad}}(x, C)<\bar{d}$ (cf Proposition 3.9) there exists an open subset $V$ of $N, \widetilde{B}\left(x^{\prime}, 1 / \epsilon_{1}-\frac{101}{100} \bar{d}\right) \subset V \subset \widetilde{B}\left(x^{\prime}, 1 / \epsilon_{1}\right)$, such that $\phi(V)$ is $\rho(H)$-invariant. The pulled-back action $\phi^{*} \rho$ on $V$ is $\tilde{\epsilon}_{1}\left(\epsilon_{1}\right)$-isometric with $\tilde{\epsilon}_{1}\left(\epsilon_{1}\right) \rightarrow 0$ as $\epsilon_{1} \rightarrow 0$. Let $A \subset \widetilde{B}\left(x^{\prime}, \frac{101}{100} \bar{d}\right)$ be a $\left(\phi^{*} \rho\right)(H)$-invariant open neighborhood of $x^{\prime}$ such that $C \subset \phi(A)$.

Now we can apply Lemma 4.1. We fix some $\zeta_{0}>0$ (which will not play a role afterwards) and choose $\epsilon_{1}^{(2)} \in\left(0, \epsilon_{1}^{(1)}\right]$ sufficiently small such that $0<\epsilon_{1} \leq \epsilon_{1}^{(2)}$ implies $\tilde{\epsilon}_{1}\left(\epsilon_{1}\right) \leq \eta\left(\frac{101}{100} \bar{d}, \zeta_{0}, H\right)=: \eta(H, \epsilon)$. The lemma yields a time slice $\left(\widehat{N}, \hat{x}^{\prime}\right)$ of a $\kappa_{0}-$ or rescaled standard solution, an isometric action $\hat{\rho}: H \curvearrowright \widehat{N}$, and a $\left(\phi^{*} \rho, \hat{\rho}\right)-$ equivariant embedding $\iota: A \hookrightarrow \hat{N}$. The latter implies that the action $\left.\phi^{*} \rho\right|_{\phi^{-1}(\bar{C})}$ is

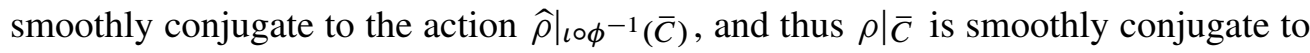

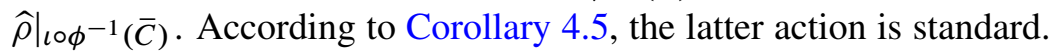

We will henceforth put $\epsilon_{1}^{(3)}(G, \epsilon):=\min \left\{\epsilon_{1}^{(2)}(H, \epsilon): H \leq G\right\}$ and assume that $0<$ $\epsilon_{1} \leq \epsilon_{1}^{(3)}$.

\section{Equivariant Ricci flow with cutoff and applications}

We will now derive our main results about smooth actions by finite groups on closed 3-manifolds. Given an action $\rho_{0}: G \curvearrowright M_{0}$, we choose a $\rho_{0}$-invariant Riemannian metric $g_{0}$ on $M_{0}$. Perelman's construction of Ricci flow with cutoff carries over to the equivariant case in a straight-forward manner, see Section 5.1, and yields an equivariant Ricci flow with cutoff defined for all times and with initial time slice $\rho_{0}: G \curvearrowright\left(M_{0}, g_{0}\right)$. Based on the fact proven in Section 4 that actions on caps in the highly curved regions near the singularities of the Ricci flow are standard, we are able to classify the actions on the time slices short before extinction and, more generally, describe the change of the actions when crossing a singular time. We then focus on the case when the initial manifold $M_{0}$ is irreducible and further on actions on elliptic and hyperbolic 3 -manifolds. Finally, we discuss actions on $\left(S^{2} \times \mathbb{R}\right)$-manifolds.

\subsection{Existence}

The construction of a Ricci flow with $(r, \delta)$-cutoff, ie with a specified way of surgery, for closed orientable 3-manifolds with arbitrary initial metrics is one of the fundamental contributions of Perelman [27]. For a detailed discussion of Ricci flows with surgery we 
refer to Kleiner and Lott [15, Sections 68-80], Morgan and Tian [21, Chapters 13-17] and Bamler [1, Chapter 7]. We will adopt the notation in [15, Section 68]. There is the following difference, however. We use the parameter $\epsilon_{1}$ (instead of $\epsilon$ ) to measure the quality of approximation of canonical neighborhoods by local models ( $\kappa_{0}$-solutions, shrinking spherical space forms or standard solutions). That is, our parameter $\epsilon_{1}$ plays the role of the parameter $\epsilon$ in [15, Lemma 59.7 and Definition 69.1].

For a Ricci flow with surgery $\left(\mathcal{M},\left(g_{t}\right)_{0 \leq t<+\infty}\right)$ there is a discrete, possibly empty or infinite, sequence of singular times $0<t_{1}<\cdots<t_{k}<\ldots$. Let $k_{\max }$ denote the number of singular times, $0 \leq k_{\max } \leq+\infty$. We denote by $M_{k}$ the orientable closed 3-manifold underlying the time slices $\mathcal{M}_{t_{k}}^{+}$and $\mathcal{M}_{t}$ for $t_{k}<t<t_{k+1}$. (We put $t_{0}:=0$ and, if $k_{\max }<+\infty$, also $t_{k_{\max }+1}:=+\infty$.)

We will only consider Ricci flows with $(r, \delta)$-cutoff. These are Ricci flows with surgery where the surgery is performed in a specific way. Let us recall how one passes at a singular time $t_{k}$ from the backward (presurgery) time slice $\mathcal{M}_{t_{k}}^{-}$to the forward (postsurgery) time slice $\mathcal{M}_{t_{k}}^{+}$; compare [15, Definition 73.1]. The manifold underlying $\mathcal{M}_{t_{k}}^{-}$is the open subset $\Omega=\left\{x \in M_{k-1}\left|\lim \sup _{t} \tau_{k}\right| R(x, t) \mid<\infty\right\}$, where $R$ is the Riemann curvature tensor. On $\Omega$ the Riemannian metrics converge smoothly to a limit metric, $g_{t} \rightarrow g_{t_{k}}^{-}$as $t \nearrow t_{k}$. Let $\rho:=\delta\left(t_{k}\right) r\left(t_{k}\right)$. In order to obtain $\mathcal{M}_{t_{k}}^{+}$, we discard all components of $\Omega$ that do not intersect $\Omega_{\rho}=\left\{x \in \Omega \mid S\left(x, t_{k}\right) \leq \rho^{-2}\right\}$. We say that a component of $M_{k-1}$ that does not intersect $\Omega_{\rho}$ goes extinct at time $t_{k}$. For volume reasons, there are only finitely many components $\Omega_{i}$ of $\Omega$ which do intersect $\Omega_{\rho}$. If a component $\Omega_{i}$ is closed then the Ricci flow smoothly extends to times after $t_{k}$ and $\Omega_{i}$ survives to $M_{k}$ without being affected by the surgery. Each noncompact component $\Omega_{i}$ has finitely many ends, and the ends are represented by $\epsilon_{1}$-horns $\mathcal{H}_{i j} \subset \Omega_{i}$. The horns are disjoint and contained in the foliated necklike region $F$ introduced in Section 3.3. The surgery is performed at $\delta\left(t_{k}\right)$-necks $\mathcal{N}_{i j} \subset \mathcal{H}_{i j}$ which are centered at points with scalar curvature $h\left(t_{k}\right)^{-2}$. The quantity $h\left(t_{k}\right)<\rho$ is given by [15, Lemma 71.1]. (The necks $\mathcal{N}_{i j}$ are in fact final time slices of strong $\delta\left(t_{k}\right)$-necks.) The horn $\mathcal{H}_{i j}$ is cut along the (with respect to the neck parametrization) central $\mathcal{F}$-leaf, ie cross-sectional sphere $S_{i j} \subset \mathcal{N}_{i j}$ and capped off by attaching a 3-ball. The region $X:=\mathcal{M}_{t_{k}}^{-} \cap \mathcal{M}_{t_{k}}^{+}$ common to backward and forward time slice is a compact 3-manifold with boundary $\partial X$ equal to the union of the surgery spheres $S_{i j}$. One may regard $X$ as a submanifold of both $M_{k-1}$ and $M_{k}$.

Let now $G$ be a finite group. A $G$-equivariant Ricci flow with surgery consists of a Ricci flow with surgery $\left(\mathcal{M},\left(g_{t}\right)_{0 \leq t<+\infty}\right)$ together with a smooth group action $\rho: G \curvearrowright \mathcal{M}$ such that $\rho$ preserves each time slice $\mathcal{M}_{t}^{ \pm}$and acts on it isometrically. Moreover, we require that $\rho$ maps static curves to static curves. The restriction of $\rho$ 
to the time slab $\mathcal{M}_{\left(t_{k}, t_{k+1}\right)}$ corresponds to a smooth action $\rho_{k}: G \curvearrowright M_{k}$ which is isometric with respect to the Riemannian metrics $g_{t_{k}}^{+}$and $g_{t}$ for $t_{k}<t<t_{k+1}$.

In [27], Perelman only discussed the nonequivariant case (ie when $G$ is trivial), but not much has to be modified to extend the discussion to the equivariant case. The usual Ricci flow without surgery on a closed 3-manifold preserves the symmetries of the initial metric (as a consequence of its uniqueness). Hence the metrics $g_{t}, t_{k}<t<t_{k+1}$, will have the same symmetries as $g_{t_{k}}^{+}$. To obtain an equivariant Ricci flow with cutoff for a given equivariant initial condition, one must only ensure that no symmetries get lost in the surgery process. Once the surgery necks are chosen equivariantly in the sense of Lemma 3.8, the surgery process as described in [15, Section 72] does preserve the existing symmetries.

The equivariant choice of surgery necks can be easily achieved. One can arrange that every $\epsilon_{1}$-horn $\mathcal{H}_{i j}$ at the surgery time $t_{k}$ is saturated with respect to the foliation $\mathcal{F}$ and that the union of the horns is $\rho$-invariant, ie the horns are permuted by the group action. Note that $H_{i j}=\operatorname{Stab}_{G}\left(\mathcal{H}_{i j}\right)$ preserves every $\mathcal{F}$-leaf in $\mathcal{H}_{i j}$. The $\delta\left(t_{k}\right)$-necks $\mathcal{N}_{i j}$ can also be chosen $\rho$-equivariantly and as $\mathcal{F}$-saturated subsets, and then $H_{i j}=\operatorname{Stab}_{G}\left(\mathcal{N}_{i j}\right)$. Using Lemma 3.8, we $\rho$-equivariantly replace the $\mathcal{N}_{i j}$ by $\left(H_{i j}, \tilde{\delta}\left(\delta\left(t_{k}\right), G\right)\right)$-necks $\widetilde{\mathcal{N}}_{i j}$ centered at the same points. The $\widetilde{\mathcal{N}}_{i j}$ have the additional property that the approximating $\tilde{\delta}$-homothetic diffeomorphisms $\phi_{i j}: S^{2}(\sqrt{2}) \times(-1 / \widetilde{\delta}, 1 / \widetilde{\delta}) \rightarrow \widetilde{\mathcal{N}}_{i j}$ can be chosen such that the pulled-back actions $\hat{\rho}_{i j}=\phi_{i j}^{*}\left(\left.\rho\right|_{H_{i j}}\right)$ on $S^{2}(\sqrt{2}) \times(-1 / \tilde{\delta}, 1 / \tilde{\delta})$ are isometric (and trivial on the interval factor). Since $\tilde{\delta}(\delta, G) \rightarrow 0$ as $\delta \rightarrow 0$, we can keep $\tilde{\delta}$ arbitrarily small by suitably decreasing $\delta$.

To glue in the surgery caps, we follow the interpolation procedure of [15, Lemma 72.24]. The surgery caps are truncated standard solutions and have the full $O(3)$-symmetry. The gluing can therefore be done equivariantly and so $\rho$ extends to an isometric action on the glued in surgery caps, that is, on the entire time slice $\mathcal{M}_{t_{k}}^{+}$. The time- $t_{k}$ Hamilton-Ivey pinching condition is satisfied on $\mathcal{M}_{t_{k}}^{+}$if $\delta$ is chosen sufficiently small.

The justification of the a priori conditions, ie the argument that for a suitable choice of the parameter functions $r, \delta:[0, \infty) \rightarrow(0, \infty)$ the canonical neighborhood condition as formulated in [15, Section 69] remains valid during the flow, is not affected by the presence of a group action; compare [15, Section 77].

One concludes (cf [27, Proposition 5.1] and [15, Proposition 77.2]) that there exists $\epsilon_{1}^{(4)}(G)>0$ such that the following holds: If $0<\epsilon_{1} \leq \epsilon_{1}^{(4)}$, then there exist positive nonincreasing functions $r, \bar{\delta}:[0, \infty) \rightarrow(0, \infty)$ such that for any normalized initial data $\rho: G \curvearrowright \mathcal{M}_{0}$ and any nonincreasing function $\delta:[0, \infty) \rightarrow(0, \infty)$ with $\delta<\bar{\delta}$, the $G$-equivariant Ricci flow with $(r, \delta)$-cutoff is defined for all times. 
We will henceforth put $\epsilon_{1}^{(5)}(G, \epsilon):=\min \left(\epsilon_{1}^{(4)}(G), \epsilon_{1}^{(3)}(G, \epsilon)\right)$ and assume that $0<$ $\epsilon_{1} \leq \epsilon_{1}^{(5)}$.

\subsection{Standard actions short before extinction}

Let $\rho$ : $G \curvearrowright \mathcal{M}$ be an equivariant Ricci flow with cutoff. We consider now the situation when some of the connected components of $M_{k-1}$ go extinct at the singular time $t_{k}$. It is known (cf [15, Section 67]) that each such component is diffeomorphic to a spherical space form, to $\mathbb{R} P^{3} \sharp \mathbb{R} P^{3}$ or to $S^{2} \times S^{1}$.

Theorem 5.1 (Extinction) Suppose that $M_{k-1}^{(1)}$ is a connected component of $M_{k-1}$ which goes extinct at the singular time $t_{k}$. Then the part $\operatorname{Stab}_{G}\left(M_{k-1}^{(1)}\right) \curvearrowright M_{k-1}^{(1)}$ of the action $\rho_{k-1}: G \curvearrowright M_{k-1}$ is standard.

We recall from Section 2.1 that an action on a spherical space form is standard if and only if it is smoothly conjugate to an isometric action, and an action on $S^{2} \times S^{1}$ or $\mathbb{R} P^{3} \sharp \mathbb{R} P^{3}$ is standard if and only if there exists an invariant Riemannian metric locally isometric to $S^{2} \times \mathbb{R}$.

Proof If $t \in\left(t_{k-1}, t_{k}\right)$ is sufficiently close to $t_{k}$, then $\mathcal{M}_{t}=\left(M_{k-1}^{(1)}, g_{t}\right)$ has everywhere high scalar curvature, $S>\frac{99}{100} r\left(t_{k}\right)^{-2} \delta\left(t_{k}\right)^{-2}$, and is therefore everywhere locally $\epsilon_{1}$-approximated by a local model, ie we have that $M_{k-1}^{(1)}=A_{0}$. (We adopt the notation of Section 3 with $\left(M_{k-1}^{(1)}, g_{t}\right)$ playing the role of $(M, g)$.)

If there exists $x \in M_{k-1}^{(1)}$ with $\widetilde{\operatorname{rad}}_{t}\left(x, M_{k-1}^{(1)}\right) \leq D$, then $\left(M_{k-1}^{(1)}, g_{t}\right)$ has strictly positive sectional curvature; compare (the end of) Section 3.4 and the assertion follows from Hamilton [12].

We may therefore assume that $M_{k-1}^{(1)}=A_{1}$ and consider the equivariant tube-cap decomposition as in Situation 1 of Section 3.5. If $F=M_{k-1}^{(1)}$, then $M_{k-1}^{(1)} \cong S^{2} \times S^{1}$ and by Corollary 2.7, $\operatorname{Stab}_{G}\left(M_{k-1}^{(1)}\right) \curvearrowright M_{k-1}^{(1)}$ is standard. Otherwise, the tube-cap decomposition has the form $M_{k-1}^{(1)}=C_{1} \cup T \cup C_{2}$ as in (3.11), and $M_{k-1}^{(1)}$ is diffeomorphic to $S^{3}, \mathbb{R} P^{3}$ or $\mathbb{R} P^{3} \sharp \mathbb{R} P^{3}$. The $\rho_{k-1}$-action of $\operatorname{Stab}_{G}\left(M_{k-1}^{(1)}\right)$ preserves the central $\mathcal{F}$-leaf of $T$ but it may switch the caps. The stabilizer $G^{\prime}:=\operatorname{Stab}_{G}\left(C_{1}\right)=\operatorname{Stab}_{G}\left(C_{2}\right)$ has index 1 or 2 in $\operatorname{Stab}_{G}\left(M_{k-1}^{(1)}\right)$. According to Proposition 4.6, the restriction of the $\rho_{k-1}\left(G^{\prime}\right)$-action to each cap $C_{i}$ is standard. With Corollary 2.6 we conclude that $\operatorname{Stab}_{G}\left(M_{k-1}^{(1)}\right) \curvearrowright M_{k-1}^{(1)}$ is standard. 


\subsection{Topological effect of surgery on group actions}

Let $\rho$ : $G \curvearrowright \mathcal{M}$ be an equivariant Ricci flow with cutoff. We describe now in general, how the actions before and after a surgery time are related to each other.

Theorem 5.2 (Topological effect of surgery) For each $k \geq 1$, the action $\rho_{k-1}$ before the surgery time $t_{k}$ is obtained from the action $\rho_{k}$ afterwards in three steps:

(i) First, one takes the disjoint union of $\rho_{k}$ with a standard action on a finite (possibly empty) union of $\mathbb{R} P^{3}$ 's. The stabilizer in $G$ of each such $\mathbb{R} P^{3}$ has a fixed point on it.

(ii) Then one forms an equivariant connected sum. The $\mathbb{R} P^{3}$ components mentioned in (i) correspond to ends of the graph associated to the connected sum. (Compare Section 2.3.)

(iii) Finally, one takes the disjoint union with a standard action on a closed (possibly empty) 3-manifold whose components are diffeomorphic to a spherical space form, to $\mathbb{R} P^{3} \sharp \mathbb{R} P^{3}$ or to $S^{2} \times S^{1}$. (These are the components going extinct at time $t_{k}$; cf Theorem 5.1.)

Proof Recall that $X=\mathcal{M}_{t_{k}}^{-} \cap \mathcal{M}_{t_{k}}^{+}$and $\partial X$ is the union of the surgery spheres $S_{i j}$. When passing from $M_{k-1}$ to $M_{k}, M_{k-1}-X$ is replaced by a union of balls $B_{i j}$ which are attached to the boundary spheres $S_{i j}$ of $X$, and the restriction of the action $\rho_{k-1}$ to $\overline{M_{k-1}-X}$ is replaced by a standard action on the union of the glued-in balls.

The restriction of $\rho_{k-1}$ to the closed components of $M_{k-1}-X$ is standard by Theorem 5.1. This gives step (iii). (Note that the proof follows the forward surgery process, so going backwards reverses the order of the steps.)

Let $Z$ denote the closure of the union of the components of $M_{k-1}-X$ with nonempty boundary. Then $Z$ is a compact manifold with boundary $\partial Z=\bigcup S_{i j}$. In order to analyze $\rho_{k-1} \mid Z$, we apply the equivariant tube-cap decomposition as in Situation 2 of Section 3.5. We choose a $\rho$-invariant family of funnels $Y_{i j} \subset \mathcal{H}_{i j}$ such that $\partial_{l} Y_{i j}=S_{i j}$ with respect to the metric $g_{t_{k}}^{-}$. That is, $Y_{i j}$ is contained in the end of $\Omega_{i}$ bounded by $S_{i j}$. Let $Y:=\bigcup Y_{i j}$.

For a time $t<t_{k}$ sufficiently close to $t_{k}$, the metric $g_{t_{k}}^{-}$is on the compact manifold $X \cup Y$ arbitrarily well approximated by $g_{t}$. Furthermore, $S(\cdot, t) \geq \frac{99}{100} \rho^{-2}$ on $Z$ and $\left(Z, g_{t}\right)$ is therefore everywhere locally $\epsilon_{1}$-approximated by a local model, ie we have that $Z-Y \subset A_{0}$. Since $Z$ has no closed component, we have in fact that $Z-Y \subset A_{1}$. According to the discussion in Section 3.5, each component of $\left(Z, g_{t}\right)$ is either an $\epsilon_{1}$-tentacle attached to one of the spheres $S_{i j}$ as in (3.13), or an $\epsilon_{1}$-tube connecting two of the spheres $S_{i j}$. 
Instead of removing the $\epsilon_{1}$-tubes and equivariantly attaching balls to the surgery spheres bounding them, we may cut the $\epsilon_{1}$-tubes along their central $\mathcal{F}$-leaves and attach balls to the resulting boundary spheres. The smooth conjugacy type of the action thus obtained is the same. Surgery on a tube corresponds to an edge of the graph of the equivariant connected sum of step (ii).

Tentacles are diffeomorphic to $\bar{B}^{3}$ or $\mathbb{R} P^{3}-B^{3}$, and Proposition 4.6 implies that the restriction of $\rho_{k-1}$ to the union of the $\epsilon_{1}$-tentacles is standard. We decompose the action $\rho_{k-1}$ as an equivariant connected sum (in the sense of Section 2.3) along the family of surgery spheres $S_{i j}$ bounding $\epsilon_{1}$-tentacles. Each tentacle contributes a summand diffeomorphic to $S^{3}$ or $\mathbb{R} P^{3}$ which corresponds to an end of the graph associated to the connected sum decomposition. In view of Corollary 2.6, the restriction of the action to the union of these summands is standard. Vice versa, when passing from $\rho_{k}$ back to $\rho_{k-1}$, the effect of replacing the surgery caps $B_{i j}$ corresponding to $\epsilon_{1}$-tentacles by the tentacles amounts to a connected sum with a standard action on a union of $S^{3}$ 's and $\mathbb{R} P^{3}$ 's, and these latter summands correspond to ends of the graph associated to the connected sum. Adding the $S^{3}$ summands does not change the smooth conjugacy type of the resulting action, and they can therefore be omitted.

This completes the proof of the theorem.

Forgetting about the $G$-action for a moment, the effect of surgery on the topology of the time slices is as follows. $M_{k-1}$ is obtained from $M_{k}$ in two steps: First, one takes connected sums of components of $M_{k}$ and, possibly, copies of $\mathbb{R} P^{3}$ and $S^{2} \times S^{1}$. Secondly, one takes the disjoint union with finitely many (possibly zero) spherical space forms and copies of $\mathbb{R} P^{3} \sharp \mathbb{R} P^{3}$ and $S^{2} \times S^{1}$. (Note that our definition of equivariant connected sum of an action allows connected sums of components with themselves. Therefore no $S^{2} \times S^{1}$ summands are needed in the statement of part (i) of Theorem 5.2.)

\subsection{The irreducible case}

Let $\rho: G \curvearrowright \mathcal{M}$ be an equivariant Ricci flow with cutoff and suppose now that the initial manifold $M_{0}$ is irreducible. Then only 3-spheres can split off and the effect of surgery on the group action is more restricted. Theorem 5.2 specializes to:

Corollary 5.3 Suppose that the orientable closed 3-manifold $M_{0}$ is connected and irreducible.

(i) If $M_{0} \cong S^{3}$, then every manifold $M_{k}$ is a union of 3-spheres (possibly empty for $k=k_{\max }$ ). The action $\rho_{k-1}$ arises from $\rho_{k}$ by first forming an equivariant connected sum and then taking the disjoint union with a standard action on a finite union of 3-spheres. 
(ii) If $M_{0} \not S^{3}$, then there exists $k_{0}, 0 \leq k_{0} \leq k_{\max }$, such that: For $0 \leq k \leq k_{0}$, the manifold $M_{k}$ has a unique connected component $M_{k}^{(0)} \cong M_{0}$, and all other components are $\cong S^{3}$. For $k_{0}<k \leq k_{\max }, M_{k}$ is a union of 3-spheres (possibly empty for $\left.k=k_{\max }\right)$.

Furthermore, for $1 \leq k \leq k_{0}$, the action $\rho_{k-1} \mid M_{k-1}^{(0)}$ is an equivariant connected sum of actions $\left.\rho_{k}\right|_{M_{k}^{\prime}}$ where $M_{k}^{\prime}$ is a $\rho_{k}$-invariant union of $M_{k}^{(0)}$ with some of the $S^{3}-$ components of $M_{k}$.

If $k_{0}<k_{\max }$, either $M_{k_{0}}^{(0)}$ goes extinct at time $t_{k_{0}+1}$ and is diffeomorphic to a spherical space form, or $M_{k_{0}}^{(0)}$ does not go extinct at time $t_{k_{0}+1}$ and is $\cong \mathbb{R} P^{3}$. In the first case, the action $\rho_{k_{0}} \mid M_{k_{0}}^{(0)}$ is standard. In the second case, it is an equivariant connected sum of the union of a standard action on $\mathbb{R} P^{3}$ with an action on a finite union of 3-spheres.

Proof According to Theorem 5.2, $M_{0}$ is for every $k$ the connected sum of the components of $M_{k}$ and possibly further closed orientable 3-manifolds (spherical space forms and copies of $S^{2} \times S^{1}$ ). Since $M_{0}$ is irreducible, $M_{k}$ can have at most one component $M_{k}^{(0)} \not S^{3}$, and this component must itself be irreducible. If $M_{k}$ contains such a component, then so does $M_{l}$ for $0 \leq l \leq k$, and we have that $M_{k}^{(0)} \cong M_{k-1}^{(0)} \cong \cdots \cong M_{0}^{(0)}=M_{0}$. Let $k_{0},-1 \leq k_{0} \leq k_{\max }$, be maximal such that $M_{k}$ has such a component $M_{k}^{(0)}$ for $0 \leq k \leq k_{0}$.

(i) Here $k_{0}=-1$ and all components of the $M_{k}$ are 3-spheres. Step (i) in Theorem 5.2 must be empty, and the components of step (iii) can only be 3 -spheres.

(ii) Now $k_{0} \geq 0$. If $1 \leq k \leq k_{0}$, then again step (i) in Theorem 5.2 must be empty, and the components of step (iii) can only be 3 -spheres. That is, $\rho_{k-1}$ arises from $\rho_{k}$ by first taking an equivariant connected sum and then taking the disjoint union with a standard action on a finite union of 3-spheres. Our assertion for $\left.\rho_{k-1}\right|_{M_{k-1}^{(0)}}$ follows.

By Theorem 5.2, a component of $M_{k}$ which does not go extinct at time $t_{k+1}$ decomposes as the connected sum (in the usual nonequivariant sense) of some components of $M_{k+1}$ and, possibly, copies of $\mathbb{R} P^{3}$ and $S^{2} \times S^{1}$. In our situation, if $k_{0}<k_{\max }$ and $M_{k_{0}}^{(0)}$ does not go extinct at time $t_{k_{0}+1}$, then $M_{k_{0}}^{(0)}$ must be diffeomorphic to $\mathbb{R} P^{3}$, because it is irreducible and $M_{k_{0}+1}$ is a union of 3 -spheres. If $M_{k_{0}}^{(0)}$ goes extinct at time $t_{k_{0}+1}$, then it must be a spherical space form, because it is irreducible; compare the first paragraph of Section 5.2. The claim concerning $\rho_{k_{0}} \mid M_{k_{0}}^{(0)}$ follows from Theorem 5.2, respectively, from Theorem 5.1.

Now we use the deep fact that on a connected closed orientable 3-manifold with finite fundamental group the Ricci flow with cutoff goes extinct for any initial metric; see Perelman [26], Colding and Minicozzi [5], and Morgan and Tian [21]. This rules 
out nonstandard actions on 3-spheres and leads to a substantial strengthening of the conclusion of the previous corollary.

Corollary 5.4 Suppose that the orientable closed 3-manifold $M_{0}$ is connected and irreducible.

(i) If $\pi_{1}\left(M_{0}\right)$ is finite, then the Ricci flow $\mathcal{M}$ goes extinct after finite time and $M_{0}$ is diffeomorphic to a spherical space form. The initial action $\rho_{0}: G \curvearrowright M_{0}$ is standard.

(ii) If $\pi_{1}\left(M_{0}\right)$ is infinite, then the Ricci flow $\mathcal{M}$ does not go extinct after finite time. Every manifold $M_{k}$ has a unique connected component $M_{k}^{(0)} \cong M_{0}$, and the other

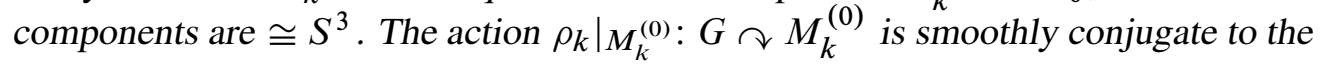
initial action $\rho_{0}: G \curvearrowright M_{0}$.

Proof If $k_{0}<k_{\max }$, then $M_{0} \cong M_{k_{0}}^{(0)}$ is a spherical space form by Corollary 5.3. On the other hand, if $\pi_{1}\left(M_{0}\right)$ is finite, then the Ricci flow $\mathcal{M}$ goes extinct in finite time and $k_{0}<k_{\max }$. Thus $k_{0}<k_{\max }$ if and only if $\pi_{1}\left(M_{0}\right)$ is finite if and only if the Ricci flow $\mathcal{M}$ goes extinct in finite time.

(i) If $M_{0} \cong S^{3}$, then Corollary 5.3 (i) and Proposition 2.10 (i) imply that $\rho_{k-1}$ is standard if $\rho_{k}$ is standard. Moreover, $M_{k_{\max }}=\varnothing$ and thus $\rho_{k_{\max }}$ is standard. It follows that $\rho_{0}$ is standard.

If $M_{0}$ is a spherical space form with nontrivial fundamental group, then $0 \leq k_{0}<k_{\max }$. Since we now know that actions on unions of 3-spheres are standard, Corollary 5.3 (ii) and Proposition 2.10 yield that $\rho_{k_{0}}$ is standard. Furthermore, $\left.\rho_{k}\right|_{M_{k}^{(0)}}$ is smoothly conjugate to $\left.\rho_{k-1}\right|_{M_{k-1}^{(0)}}$ for $1 \leq k \leq k_{0}$. Hence $\rho_{0}$ is standard.

(ii) Now $k_{0}=k_{\max }$. As in case (i), Corollary 5.3 (ii) and Proposition 2.10 yield that $\rho_{k} \mid M_{k}^{(0)}$ is smoothly conjugate to $\rho_{k-1} \mid M_{k-1}^{(0)}$ for $1 \leq k \leq k_{\max }$.

\subsection{Applications to actions on elliptic and hyperbolic 3-manifolds}

We prove now our main results.

Theorem E (Actions on elliptic manifolds are standard.) Any smooth action by a finite group on an elliptic 3-manifold is smoothly conjugate to an isometric action.

Proof Let $\rho_{0}: G \curvearrowright M_{0}$ be a smooth action by a finite group on a connected elliptic 3manifold. We recall that elliptic 3-manifolds are orientable. There exists an equivariant Ricci flow with cutoff $\rho: G \curvearrowright \mathcal{M}$ such that $\rho_{0}$ is the given action. (The $\rho_{0}$-invariant initial Riemannian metric $g_{0}$ is different from the a priori given spherical metric on $M_{0}$ 
unless the latter is already $\rho_{0}$-invariant in which case there is nothing to prove.) By Corollary 5.4 (i), the action $\rho_{0}$ is standard, ie there exists a $\rho_{0}$-invariant spherical metric $g_{\text {sph }}$ on $M_{0}$.

As mentioned in Section 2.1 already, any two diffeomorphic elliptic 3-manifolds are isometric. Thus, if $g_{\mathrm{sph}}^{\prime}$ is an a priori given spherical metric on $M_{0}$, then there exists a diffeomorphism $c$ of $M_{0}$ such that $c^{*} g_{\mathrm{sph}}=g_{\mathrm{sph}}^{\prime}$. Then the action $c^{*} \rho_{0}$ is isometric with respect to $g_{\mathrm{sph}}^{\prime}$.

Theorem H (Actions on closed hyperbolic manifolds are standard.) Any smooth action by a finite group on a closed hyperbolic 3-manifold is smoothly conjugate to an isometric action.

Proof Let $\rho_{0}: G \curvearrowright M_{0}$ be a smooth action by a finite group on a closed connected hyperbolic 3-manifold.

For a hyperbolic metric on $M_{0}$ there exists by Mostow rigidity [22] a unique isometric action $\rho_{\text {isom }}: G \curvearrowright M_{0}$ such that $\rho_{0}(\gamma)$ is homotopic to $\rho_{\text {isom }}(\gamma)$ for every $\gamma \in G$. We need to show that the actions $\rho_{0}$ and $\rho_{\text {isom }}$ are smoothly conjugate.

We assume first that $M_{0}$ is orientable. There exists an equivariant Ricci flow with cutoff $\rho: G \curvearrowright \mathcal{M}$ such that $\rho_{0}$ is the given action. By Corollary 5.4 (ii), $\mathcal{M}$ does not go extinct in finite time, every manifold $M_{k}$ has a unique component $M_{k}^{(0)} \cong M_{0}$ and $\left.\rho_{k}\right|_{M_{k}^{(0)}}$ is smoothly conjugate to $\rho_{0}$.

We will now use the analysis of the long time behavior of the Ricci flow with cutoff, see [15, Sections 87-92]. Since $M_{0}$ is not a graph manifold, the thick parts of the time slices $\mathcal{M}_{t}$ cannot be empty for large $t$; $\operatorname{cf}[15$, Sections 89 and 92] and [20, Theorem 0.2]. Therefore the collection of complete finite-volume hyperbolic 3-manifolds, which approximate the thick parts of the $\mathcal{M}_{t}$ as described in [15, Proposition 90.1], is nonempty. Furthermore, it can only consist of one closed connected hyperbolic 3manifold $H$. This follows from the $\pi_{1}$-injectivity of the approximating maps (compare [15, Proposition 91.2]) and one uses, that $M_{k}-M_{k}^{(0)}$ is a union of 3-spheres and $M_{k}^{(0)}$ contains no incompressible 2-torus.

Suppose that $T_{0}<+\infty$ and the nonincreasing function $\alpha:\left[T_{0}, \infty\right) \rightarrow(0, \infty)$ with $\lim _{t \rightarrow \infty} \alpha(t)=0$ are as in [15, Proposition 90.1]. Since $H$ is closed, the conclusion of [15, Proposition 90.1] yields: There exists $T_{1} \in\left[T_{0}, \infty\right)$ such that for any time $t \geq T_{1}$ there is an $\alpha(t)$-homothetic embedding

$$
f_{t}: H \rightarrow \mathcal{M}_{t}
$$


which is a diffeomorphism onto a connected component of $\mathcal{M}_{t}$. Moreover, $f_{t}$ depends smoothly on $t$. Note that the image of $f_{t}$ avoids the regions where surgeries take place, because on it the scalar curvature is negative. Thus, if $t=t_{k} \geq T_{1}$ is a singular time, then $\operatorname{im}\left(f_{t}\right)$ is a closed component of $\mathcal{M}_{t}^{-} \cap \mathcal{M}_{t}^{+}$which is not affected by surgeries.

All other components of the time slices $\mathcal{M}_{t}, t \geq T_{1}$, are 3-spheres. These go extinct in finite time. Hence there exists $T_{2} \in\left[T_{1}, \infty\right)$ such that $f_{t}$ is a diffeomorphism onto $\mathcal{M}_{t}$ for $t \geq T_{2}$. We conclude that, up to scaling, $\mathcal{M}_{t}$ converges smoothly to $H$. More precisely, one has

$$
\frac{1}{2 t} f_{t}^{*} g_{t} \stackrel{\mathcal{C}^{\infty}}{\longrightarrow} g_{H}
$$

as $t \rightarrow \infty$ (compare (1) of [15, Proposition 90.1]) where we normalize the hyperbolic metric $g_{H}$ on $H$ to have sectional curvature $\equiv-1$.

Let us denote $\rho_{t}:=\left.\rho\right|_{\mathcal{M}_{t}}$. The pulled-back actions $f_{t}^{*} \rho_{t}$ on $H, t \geq T_{2}$, are smoothly conjugate to each other. Since they also become increasingly isometric $(\alpha(t) \rightarrow 0)$, Arzelà-Ascoli implies that for any sequence $\left(t_{n}\right), T_{2} \leq t_{n} \nearrow \infty$, the actions $f_{t_{n}}^{*} \rho_{t_{n}}$ subconverge smoothly to an isometric action $\bar{\rho}: G \curvearrowright H$. (It must coincide with the unique isometric action homotopic to the $f_{t}^{*} \rho_{t}$ given by Mostow rigidity; compare our remark at the beginning of the proof.) Thus for large $n$ the action $f_{t_{n}}^{*} \rho_{t_{n}}$ is a $\mathcal{C}^{\infty}$-small perturbation of the isometric limit action. Using the stability property of smooth actions (of compact Lie groups on closed manifolds) that sufficiently $\mathcal{C}^{1}$-small perturbations are smoothly conjugate (see Palais [24] and Grove-Karcher [10]), it follows that the $f_{t}^{*} \rho_{t}$ are smoothly conjugate to $\bar{\rho}$ for $t \geq T_{2}$. With Corollary 5.4 (ii) we conclude that $\rho_{0} \cong \rho_{t} \cong f_{t}^{*} \rho_{t} \cong \bar{\rho}$, ie there exists a $\rho_{0}$-invariant hyperbolic metric $g_{\text {hyp }}$ on $M_{0}$.

Suppose now that $M_{0}$ is not orientable and consider the orientable double covering $\widehat{M}_{0} \rightarrow M_{0}$. Then $M_{0}$ is the quotient of $\hat{M}_{0}$ by a smooth orientation reversing involution $\iota$. The action $\rho_{0}$ lifts to an action $\hat{\rho}_{0}: \widehat{G} \curvearrowright \widehat{M}_{0}$ of an index two extension $\widehat{G}$ of $G$. The nontrivial element in the kernel of the natural projection $\widehat{G} \rightarrow G$ is $\iota$. By the above, there exists a $\hat{\rho}_{0}$-invariant hyperbolic metric $\hat{g}_{\text {hyp }}$ on $\hat{M}_{0}$. This metric descends to a $\rho_{0}$-invariant hyperbolic metric $g_{\text {hyp }}$ on $M_{0}$. This finishes the proof that the action $\rho_{0}$ is geometric.

Finally, if $g_{\text {hyp }}^{\prime}$ is an a priori given hyperbolic metric on $M_{0}$ (unrelated to the action $\rho_{0}$ ), then Mostow rigidity yields a diffeomorphism $c$ of $M_{0}$ such that $c^{*} g_{\text {hyp }}=g_{\text {hyp }}^{\prime}$. Thus the action $\rho_{0}$ can be smoothly conjugated to the $g_{\text {hyp }}^{\prime}$-isometric action $c^{*} \rho_{0}$.

The argument for proving Theorem $\mathrm{H}$ can be extended to the case of actions on hyperbolic 3-manifolds with cusps. Let $M$ be a compact 3-manifold with nonempty 
boundary which admits a hyperbolic structure, ie whose interior admits a complete hyperbolic metric $g_{\text {hyp }}$ with finite volume. Then the ends of $\left(\operatorname{int}(M), g_{\text {hyp }}\right)$ are cusps and the boundary components of $M$ are tori or Klein bottles.

Theorem 5.5 (Actions on hyperbolic manifolds with cusps are standard.) Any smooth action $\rho: G \curvearrowright M$ by a finite group is smoothly conjugate to the restriction of an isometric action on $\left(\operatorname{int}(M), g_{\text {hyp }}\right)$ to a compact submanifold obtained from truncating the cusps.

Proof As in the case of Theorem $\mathrm{H}$ the nonorientable case can be reduced to the orientable one. We assume therefore that $M$ is orientable and connected.

We denote by $\widehat{M}_{0}$ the closed 3-manifold obtained from doubling $M$ along the boundary. The action $\rho$ generates together with the natural involution $\iota: \hat{M}_{0} \rightarrow \widehat{M}_{0}$ the action $\hat{\rho}_{0}: \widehat{G}=G \times \mathbb{Z} / 2 \mathbb{Z} \curvearrowright \widehat{M}_{0}$. (We will identify $G$ with the subgroup $G \times\{0\} \subset \widehat{G}$.)

Let $\hat{\rho}: \widehat{G} \curvearrowright\left(\widehat{\mathcal{M}},\left(\widehat{g}_{t}\right)\right)$ be an equivariant Ricci flow with cutoff whose initial action $\hat{\rho}_{0}$ is the given action. Since $\widehat{M}_{0}$ is irreducible and has infinite fundamental group, the flow does not go extinct in finite time by Corollary 5.4. For each $t \geq 0$ there is a unique connected component $\hat{\mathcal{M}}_{t}^{(0)}$ of $\widehat{\mathcal{M}}_{t}^{+}$(ie of $\hat{\mathcal{M}}_{t}$ if $t$ is a regular time) which is ( $\hat{\rho}_{t}, \hat{\rho}_{0}$ )-equivariantly diffeomorphic to $\widehat{M}_{0}$. We must take into account the possibility that surgeries occur arbitrarily late.

Again $\widehat{M}_{0}$ is not a graph manifold and hyperbolic components must form in the thick part of the time slice $\hat{\mathcal{M}}_{t}$. Since they are incompressible, they can only appear in the component $\hat{\mathcal{M}}_{t}^{(0)} \cong \widehat{M}_{0}$.

We consider one of these hyperbolic components. To adapt the formulation of [15, Proposition 90.1] to our purposes, we use the following notation: Given a complete Riemannian manifold $N$ and a real number $r>0$, we denote by $N_{r}:=\{x \in N: \operatorname{inj}(x) \geq r\}$ its $r-$ thick part in the injectivity radius sense. Then according to [15, Proposition 90.1] there exist a number $T_{0} \geq 0$, a (continuous) nonincreasing function $\beta:\left[T_{0}, \infty\right) \rightarrow(0, \infty)$ with $\lim _{t \rightarrow \infty} \beta(t)=0$, an orientable connected complete noncompact hyperbolic 3 -manifold $\left(H, g_{H}\right)$ with finite volume, and a smooth family of $\beta(t)$-homothetic embeddings

$$
f_{t}: H_{\beta(t)} \hookrightarrow \hat{\mathcal{M}}_{t}^{(0)}, \quad t \geq T_{0},
$$

avoiding the surgery regions and such that $\frac{1}{2 t} f_{t}^{*} g_{t} \rightarrow g_{H}$ smoothly as $t \rightarrow \infty$. We may assume that $\beta$ is so small that $H_{\beta(t)}$ is a compact codimension-zero submanifold (a compact core) of $H$ bounded by horospherical 2-tori. (Note that the horospherical cross sections of the cusps of $H$ are tori because $H$ is orientable. Therefore the injectivity 
radius is constant along these cross sections.) Furthermore, the embeddings $f_{t}$ are $\pi_{1}$-injective.

In view of the topological structure of $\hat{M}_{0}$, the uniqueness of the torus decomposition (see eg Hatcher [13, Theorem 1.9]) implies that $\operatorname{im}\left(f_{t}\right)$ corresponds to one of the hyperbolic components $M$ and $\iota M$ of $\hat{M}_{0}$ and is $G$-invariant up to isotopy. More precisely, for $0<\beta<\beta\left(T_{0}\right)$ and sufficiently large $t \geq T(\beta)$, the family of incompressible tori $f_{t}\left(\partial H_{\beta}\right)$ is isotopic to the family of "separating" tori $\operatorname{Fix}\left(\hat{\rho}_{t}(\iota)\right) \cap \hat{\mathcal{M}}_{t}^{(0)}$. Moreover, for $\hat{\gamma} \in \hat{G}$ the translate $\hat{\rho}_{t}(\hat{\gamma})\left(f_{t}\left(H_{\beta}\right)\right)$ is isotopic to $f_{t}\left(H_{\beta}\right)$ if and only if $\hat{\gamma} \in G$. Here we use the fact that $M$ and $\iota M$ are not isotopic to each other in $\widehat{M}_{0}$.

We argue next that the almost hyperbolic components $f_{t}\left(H_{\beta}\right)$ are essentially $G-$ invariant and not only invariant up to isotopy.

Let $0<\beta_{1}<\beta_{2}<\beta\left(T_{0}\right)$. Then for $\hat{\gamma} \in \widehat{G}$ and sufficiently large $t \geq T\left(\beta_{1}, \beta_{2}\right)$ the submanifold $\hat{\rho}_{t}(\hat{\gamma})\left(f_{t}\left(H_{\beta_{2}}\right)\right)$ is disjoint from the collection of incompressible boundary tori $f_{t}\left(\partial H_{\beta_{1}}\right)$ because the injectivity radius is strictly smaller along the latter. Since $H_{\beta_{2}}$ is connected, it follows that $\hat{\rho}_{t}(\hat{\gamma})\left(f_{t}\left(H_{\beta_{2}}\right)\right)$ is either contained in or disjoint from $f_{t}\left(H_{\beta_{1}}\right)$. In the first case $\hat{\rho}_{t}(\hat{\gamma})\left(f_{t}\left(H_{\beta_{2}}\right)\right)$ is isotopic to $f_{t}\left(H_{\beta_{1}}\right)$ and in the second case to its complement in $\hat{\mathcal{M}}_{t}^{(0)}$. It follows that $\hat{\rho}_{t}(\hat{\gamma})\left(f_{t}\left(H_{\beta_{2}}\right)\right) \subset f_{t}\left(H_{\beta_{1}}\right)$ if $\hat{\gamma} \in G$ and $\hat{\rho}_{t}(\hat{\gamma})\left(f_{t}\left(H_{\beta_{2}}\right)\right) \cap f_{t}\left(H_{\beta_{2}}\right)=\varnothing$ otherwise. In particular, $\hat{\rho}_{t}(\hat{\gamma})\left(f_{t}\left(H_{\beta_{2}}\right)\right)$ does not intersect the fixed point set of $\hat{\rho}_{t}(\iota)$ and is strictly contained in one of the halves of $\hat{\mathcal{M}}_{t}^{(0)}$ corresponding to $M$ and $\iota M$. As a consequence, we have for $0<\beta_{1}<\beta_{2}<\beta_{3}<\beta\left(T_{0}\right)$ and sufficiently large $t \geq T\left(\beta_{1}, \beta_{2}, \beta_{3}\right)$ that $f_{t}\left(H_{\beta_{3}}\right) \subset \hat{\rho}_{t}(\gamma)\left(f_{t}\left(H_{\beta_{2}}\right)\right) \subset f_{t}\left(H_{\beta_{1}}\right)$ for all $\gamma \in G$. We see that, modulo the identification $\hat{\mathcal{M}}_{t}^{(0)} \cong \widehat{M}_{0}$, there is an essentially $\rho$-invariant hyperbolic component forming inside $M$ and isotopic to $M$.

This enables us to pull back the actions $\left.\hat{\rho}_{t}\right|_{G}$ to almost isometric $G$-actions on large regions of $H$. Indeed, by the above there exists a sequence of times $t_{n} \nearrow \infty$ and $\hat{\rho}_{t_{n}}(G)-$ invariant open subsets $U_{n} \subset \operatorname{im}\left(f_{t_{n}}\right)$ with $f_{t_{n}}^{-1}\left(U_{n}\right) \nearrow H$. The pulled-back actions $f_{t_{n}}^{*}\left(\left.\hat{\rho}_{t_{n}}\right|_{G}\right)$ are defined (at least) on $f_{t_{n}}^{-1}\left(U_{n}\right)$ and become more and more isometric. As in the proof of Theorem $\mathrm{H}$, it follows using Arzelà-Ascoli that after passing to a subsequence the actions $f_{t_{n}}^{*}\left(\left.\hat{\rho}_{t_{n}}\right|_{G}\right)$ converge smoothly (and locally uniformly) to an isometric action $\bar{\rho}: G \curvearrowright H$, ie for large $n$ the action $f_{t_{n}}^{*}\left(\left.\hat{\rho}_{t_{n}}\right|_{G}\right)$ is a $\mathcal{C}^{\infty}$-small perturbation of $\bar{\rho}$. Applying Grove-Karcher [10] again, there exist $f_{t_{n}}^{*}\left(\left.\hat{\rho}_{t_{n}}\right|_{G}\right)$-invariant open subsets $V_{n} \subset f_{t_{n}}^{-1}\left(U_{n}\right)$ with $V_{n} \nearrow H$ and smooth embeddings $c_{n}: V_{n} \rightarrow H$ with $c_{n} \rightarrow \mathrm{id}_{H}$ such that $\bar{\rho}(\gamma) \circ c_{n}=c_{n} \circ f_{t_{n}}^{*}\left(\hat{\rho}_{t_{n}}(\gamma)\right)$ on $V_{n}$ for $\gamma \in G$; compare the end of the proof of Lemma 4.1. In particular, we obtain that for small $\beta \in\left(0, \beta\left(T_{0}\right)\right)$ and sufficiently large $n \geq n(\beta)$ the $G$-actions $f_{t_{n}}^{*} \hat{\rho}_{t_{n}} \mid c_{n}^{-1}\left(H_{\beta}\right)$ and $\left.\bar{\rho}\right|_{H_{\beta}}$ are smoothly conjugate. This implies that there exists a $(\bar{\rho}, \rho)$-equivariant embedding $\psi: H_{\beta} \hookrightarrow$ $\operatorname{int}(M)$. 
The complementary region $M-\psi\left(\operatorname{int}\left(H_{\beta}\right)\right)$ is a union of copies of $T^{2} \times[0,1]$. The restriction of the action $\rho$ to these components is standard according to Meeks and Scott [17, Theorem 8.1]. (A smooth finite group action on $\Sigma \times[0,1], \Sigma$ a closed surface, is conjugate to a product action.) We therefore can modify $\psi$ to be a $(\bar{\rho}, \rho)$-equivariant diffeomorphism $H_{\beta} \rightarrow \operatorname{int}(M)$.

The hyperbolic manifolds $\left(H, g_{H}\right)$ and $\left(\operatorname{int}(M), g_{\text {hyp }}\right)$ are isometric by MostowPrasad rigidity $[22 ; 28]$. This completes the proof of the theorem.

\subsection{Actions on $\left(S^{2} \times \mathbb{R}\right)-$ manifolds}

Let $\rho: G \curvearrowright \mathcal{M}$ be an equivariant Ricci flow with cutoff and suppose that $M_{0}$ is a closed connected 3-manifold admitting an $\left(S^{2} \times \mathbb{R}\right)$-structure, that is a Riemannian metric locally isometric to $S^{2} \times \mathbb{R}$. Also in this case, the Ricci flow goes extinct in finite time for any initial metric; see Perelman [26] and Colding and Minicozzi [5].

Theorem 5.6 The initial action $\rho_{0}: G \curvearrowright M_{0}$ is standard.

Proof (i) Suppose that $M_{0} \cong S^{2} \times S^{1}$.

If $\mathcal{M}$ goes extinct at time $t_{1}$, then $\rho_{0}$ is standard by Theorem 5.1. We assume therefore that $\mathcal{M}$ does not go extinct at time $t_{1}$. Since $S^{2} \times S^{1}$ is prime, and in particular does not have $\mathbb{R} P^{3}$ as a connected summand, Theorem 5.2 yields that $\rho_{0}$ is an equivariant connected sum of $\rho_{1}$, respectively, $\rho_{1}$ is an equivariant connected sum decomposition of $\rho_{0}$ in the sense of Section 2.3.

We first consider the case when the family of 2-spheres, along which $M_{0}$ is decomposed, contains a nonseparating sphere. Then all components of $M_{1}$ are 3-spheres and the action $\rho_{1}$ is standard by Theorem E. Using the notation of Section 2.3, the graph $\Gamma$ associated to the connected sum decomposition of $M_{0}$ is homotopy equivalent to a circle. Equivalently, it contains a unique embedded cycle $\gamma$ (which may be a loop) which consists precisely of the nonseparating edges. We divide the family $\mathcal{P}$ of two point subsets into the subfamily $\mathcal{P}_{1}$ corresponding to the edges of $\gamma$ and its complement $\mathcal{P}_{2}$. Let $M_{1}^{\prime}$ be the union of the components of $M_{1}$ corresponding to the vertices of $\gamma$. Note that $M_{1}^{\prime}$ and the $\mathcal{P}_{i}$ are $\rho_{1}$-invariant due to the uniqueness of $\gamma$. We have $\rho_{0} \cong\left(\rho_{1}\right)_{\mathcal{P}} \cong\left(\left(\rho_{1}\right)_{\mathcal{P}_{2}}\right)_{\mathcal{P}_{1}}$. Lemma 2.9 yields as in the proof of Proposition 2.10 that $\left.\left(\rho_{1}\right)_{\mathcal{P}_{2}} \cong \rho_{1}\right|_{M_{1}^{\prime}}$. So $\rho_{0} \cong\left(\left.\rho_{1}\right|_{M_{1}^{\prime}}\right)_{\mathcal{P}_{1}}$. We may therefore assume without loss of generality that $\gamma=\Gamma, M_{1}^{\prime}=M_{1}$ and $\mathcal{P}_{1}=\mathcal{P}$. Let us denote the component 3 -spheres of $M_{1}$ by $S_{i}^{3}$ parametrized by the index set $\mathbb{Z} / l \mathbb{Z}, l \geq 1$, and the cyclic numbering chosen so that $x_{i} \in S_{i}^{3}$ and $y_{i} \in S_{i+1}^{3}$. We observe that $G_{i}$ must fix also $y_{i-1}$ and $x_{i+1}$. (Recall the definition of $G_{i}$ from the beginning 
of Section 2.3.) So $G_{i-1} \supseteq G_{i} \subseteq G_{i+1}$ and thus $G_{1}=\cdots=G_{l}$. The $\rho_{1}$-invariant spherical metric on $M_{1}$ may be arranged so that $x_{i}$ and $y_{i-1}$ are antipodal for all $i$. When performing the equivariant connected sum operation to obtain $\rho_{0}$ from $\rho_{1}$ we choose, in a $\rho_{1}$-equivariant way, Riemannian metrics on the $S_{i}^{3}-B_{r}\left(x_{i}\right)-B_{r}\left(y_{i}\right)$ isometric to $S^{2} \times[-1,1]$. This yields a $\rho_{0}$-invariant metric on $M_{0}$ locally isometric to $S^{2} \times \mathbb{R}$. Thus $\rho_{0}$ is standard in this case, too.

Suppose now that the family of $2-$ spheres, along which $M_{0}$ is decomposed, consists only of separating spheres. Then the graph $\Gamma$ is a tree. Moreover, $M_{1}$ contains a unique component $M_{1}^{(0)} \cong S^{2} \times S^{1}$ and all other components are 3 -spheres. The action on the union of 3-spheres is standard according to Theorem E. One proceeds as in the proof of Proposition 2.10 (ii) and concludes that $\left.\rho_{1}\right|_{M_{1}^{(0)}}$ is smoothly conjugate to $\rho_{0}$.

Let $k \geq 0$ be maximal such that $M_{k}$ contains a component $M_{k}^{(0)} \cong S^{2} \times S^{1}$. The above argument shows that $\rho_{k} \mid M_{k}^{(0)}$ is standard and, using induction, that $\left.\rho_{0} \cong \rho_{k}\right|_{M_{k}^{(0)}}$. It follows that $\rho_{0}$ is standard.

(ii) Now suppose that $M_{0} \not S^{2} \times S^{1}$. In order to reduce to the $S^{2} \times S^{1}$-case we argue as follows; compare the end of the proof of Theorem $\mathrm{H}$ in Section 5.5 above.

There exists a two-sheeted covering $\hat{M}_{0} \rightarrow M_{0}$ such that $\widehat{M}_{0} \cong S^{2} \times S^{1}$. Indeed, inside the deck transformation group $\operatorname{Deck}\left(M_{0}\right) \subset \operatorname{Isom}\left(S^{2} \times \mathbb{R}\right)$ we consider the subgroup $\operatorname{Deck}\left(M_{0}\right) \cap \operatorname{Isom}_{o}\left(S^{2} \times \mathbb{R}\right)$ consisting of the deck transformations which preserve the orientations of both the $S^{2}$ - and the $\mathbb{R}$-factor. Since the deck action $\operatorname{Deck}\left(M_{0}\right) \curvearrowright S^{2} \times \mathbb{R}$ is free, this subgroup has index at most two and it is the deck transformation group $\operatorname{Deck}\left(\widehat{M}_{0}\right)$ for a covering $\widehat{M}_{0} \rightarrow M_{0}$. Clearly, $\widehat{M}_{0} \cong S^{2} \times S^{1}$ and the covering is two-sheeted. (There are only three closed connected $\left(S^{2} \times \mathbb{R}\right)-$ manifolds besides $S^{2} \times S^{1}$, namely $\mathbb{R} P^{3} \sharp \mathbb{R} P^{3}, \mathbb{R} P^{2} \times S^{1}$ and the nonorientable $S^{2}$-bundle $S^{2} \tilde{\times} S^{1}$, and these are double covered by $S^{2} \times S^{1}$; compare Scott [29, page 457].)

Diffeomorphisms of $M_{0}$ lift to diffeomorphisms of the universal cover $S^{2} \times \mathbb{R}$ which normalize $\operatorname{Deck}\left(M_{0}\right)$. Let $\operatorname{Diff}_{++}\left(S^{2} \times \mathbb{R}\right)$ denote the index four normal subgroup of $\operatorname{Diff}\left(S^{2} \times \mathbb{R}\right)$ consisting of those diffeomorphisms which preserve the ends and act trivially on $H_{2}\left(S^{2} \times \mathbb{R}, \mathbb{Z}\right) \cong \mathbb{Z}$. Note that $\operatorname{Isom}_{o}\left(S^{2} \times \mathbb{R}\right)=\operatorname{Isom}\left(S^{2} \times \mathbb{R}\right) \cap$ $\operatorname{Diff}_{++}\left(S^{2} \times \mathbb{R}\right)$. Thus the normalizer of $\operatorname{Deck}\left(M_{0}\right)$ in $\operatorname{Diff}\left(S^{2} \times \mathbb{R}\right)$ also normalizes $\operatorname{Deck}\left(\hat{M}_{0}\right)=\operatorname{Deck}\left(M_{0}\right) \cap \operatorname{Diff}_{++}\left(S^{2} \times \mathbb{R}\right)$. Hence diffeomorphisms of $M_{0}$ lift to diffeomorphisms of $\hat{M}_{0}$, and the action $\rho_{0}$ lifts to an action $\hat{\rho}_{0}: \widehat{G} \curvearrowright \hat{M}_{0}$ of an index two extension $\widehat{G}$ of $G$. By part (i) of the proof there exists a $\hat{\rho}_{0}$-invariant $\left(S^{2} \times \mathbb{R}\right)-$ structure on $\hat{M}_{0}$. It descends to a $\rho_{0}$-invariant $\left(S^{2} \times \mathbb{R}\right)$-structure on $M_{0}$. 


\section{References}

[1] R Bamler, Ricci flow with surgery, PhD thesis, München (2007) Available at http:// www . math.princeton. edu/ rbamler/Ricci-flow-with-surgery . pdf

[2] M Boileau, B Leeb, J Porti, Geometrization of 3-dimensional orbifolds, Ann. of Math. (2) 162 (2005) 195-290 MR2178962

[3] E J Brody, The topological classification of the lens spaces, Ann. of Math. (2) 71 (1960) 163-184 MR0116336

[4] J Cheeger, D Gromoll, On the structure of complete manifolds of nonnegative curvature, Ann. of Math. (2) 96 (1972) 413-443 MR0309010

[5] T H Colding, W P Minicozzi, II, Estimates for the extinction time for the Ricci flow on certain 3-manifolds and a question of Perelman, J. Amer. Math. Soc. 18 (2005) 561-569 MR2138137

[6] T H Colding, W P Minicozzi, II, Width and finite extinction time of Ricci flow, Geom. Topol. 12 (2008) 2537-2586

[7] J Dinkelbach, Equivariant Ricci flow with surgery, $\mathrm{PhD}$ thesis, München (2008) Available at http://edoc.ub.uni-muenchen.de/9136/1/

[8] D B A Epstein, Curves on 2-manifolds and isotopies, Acta Math. 115 (1966) 83-107 MR0214087

[9] D Gromoll, W Meyer, On complete open manifolds of positive curvature, Ann. of Math. (2) 90 (1969) 75-90 MR0247590

[10] K Grove, H Karcher, How to conjugate $C^{1}$-close group actions, Math. Z. 132 (1973) 11-20 MR0356104

[11] K Grove, K Shiohama, A generalized sphere theorem, Ann. Math. (2) 106 (1977) 201-211 MR0500705

[12] R S Hamilton, Three-manifolds with positive Ricci curvature, J. Differential Geom. 17 (1982) 255-306 MR664497

[13] A Hatcher, Notes on basic 3-manifold topology (2000) Available at http:// www. math. cornell. edu/ hatcher/3M/3Mdownloads.html

[14] M W Hirsch, S Smale, On involutions of the 3-sphere, Amer. J. Math. 81 (1959) 893-900 MR0111044

[15] B Kleiner, J Lott, Notes on Perelman's papers, Geom. Topol. 12 (2008) 2587-2855

[16] G R Livesay, Involutions with two fixed points on the three-sphere, Ann. of Math. (2) 78 (1963) 582-593 MR0155323

[17] W H Meeks, III, P Scott, Finite group actions on 3-manifolds, Invent. Math. 86 (1986) 287-346 MR856847 
[18] W H Meeks, III, S-T Yau, Group actions on $\mathbf{R}^{3}$, from: "The Smith conjecture (New York, 1979)", Pure Appl. Math. 112, Academic Press, Orlando, FL (1984) 167-179 MR758468

[19] W Meyer, Toponogov's theorem and applications, Lecture notes, Trieste (1989) Available at http://wwwmath.uni-muenster.de/u/meyer/publications/ toponogov.html

[20] J Morgan, G Tian, Completion of the proof of the Geometrization Conjecture arXiv: 0809.4040

[21] J Morgan, G Tian, Ricci flow and the Poincaré conjecture, Clay Math. Monogr. 3, Amer. Math. Soc. (2007) MR2334563

[22] GD Mostow, Quasi-conformal mappings in n-space and the rigidity of hyperbolic space forms, Inst. Hautes Études Sci. Publ. Math. (1968) 53-104 MR0236383

[23] J Munkres, Differentiable isotopies on the 2-sphere, Michigan Math. J. 7 (1960) 193-197 MR0144354

[24] R S Palais, Equivalence of nearby differentiable actions of a compact group, Bull. Amer. Math. Soc. 67 (1961) 362-364 MR0130321

[25] G Perelman, The entropy formula for the Ricci flow and its geometric applications arXiv:math.DG/0211159

[26] G Perelman, Finite extinction time for the solutions to the Ricci flow on certain threemanifolds arXiv:math.DG/0307245

[27] G Perelman, Ricci flow with surgery on three-manifolds arXiv:math.DG/0303109

[28] G Prasad, Strong rigidity of Q-rank 1 lattices, Invent. Math. 21 (1973) 255-286 MR0385005

[29] P Scott, The geometries of 3-manifolds, Bull. London Math. Soc. 15 (1983) 401-487 MR705527

[30] J-P Serre, Linear representations of finite groups, Graduate Texts in Math. 42, Springer, New York (1977) MR0450380 Translated from the second French edition by L L Scott

[31] W P Thurston, Three-manifolds with symmetry, Preprint

[32] W P Thurston, Three-dimensional manifolds, Kleinian groups and hyperbolic geometry, Bull. Amer. Math. Soc. (N.S.) 6 (1982) 357-381 MR648524

[33] W P Thurston, Three-dimensional geometry and topology. Vol. 1, Princeton Math. Ser. 35, Princeton Univ. Press (1997) MR1435975

Mathematisches Institut der LMU

Theresienstr. 39, 80333 München, Germany

dinkelbach@mathematik. uni-muenchen.de, b.l@lmu.de

Proposed: Dave Gabai

Seconded: Colin Rourke, Tobias Colding

Received: 7 July 2008

Revised: 9 January 2009 